\title{
ON APPROXIMATIONS OF SMALL JUMPS OF SUBORDINATORS WITH PARTICULAR EMPHASIS ON A DICKMAN-TYPE LIMIT
}

\author{
SHAI COVO, ${ }^{*}$ Bar Ilan University
}

\begin{abstract}
Let $X$ be a pure-jump subordinator (i.e. nondecreasing Lévy process with no drift) with infinite Lévy measure, let $X_{\varepsilon}$ be the sum of jumps not exceeding $\varepsilon$, and let $\mu(\varepsilon)=\mathrm{E}\left[X_{\varepsilon}(1)\right]$. We study the question of weak convergence of $X_{\varepsilon} / \mu(\varepsilon)$ as $\varepsilon \downarrow 0$, in terms of the limit behavior of $\mu(\varepsilon) / \varepsilon$. The most interesting case reduces to the weak convergence of $X_{\varepsilon} / \varepsilon$ to a subordinator whose marginals are generalized Dickman distributions; we give some necessary and sufficient conditions for this to hold. For a certain significant class of subordinators for which the latter convergence holds, and whose most prominent representative is the gamma process, we give some detailed analysis regarding the convergence quality (in particular, in the context of approximating $X$ itself). This paper completes, in some respects, the study made by Asmussen and Rosiński (2001).
\end{abstract}

Keywords: Subordinator; Lévy process; small jumps; weak convergence; Dickman distribution; gamma process

2000 Mathematics Subject Classification: Primary 60G51

Secondary 60F05; 60F17

\section{Preliminaries and motivation}

In this section we provide most of the basic notation, concepts, and facts that will be used in this paper, as well as a considerable amount of motivation. An elementary familiarity with the theory of Lévy processes is presupposed. For a detailed account of the theory, we refer the reader to the classical monograph of Sato [14].

Throughout this paper, $X=\{X(t): t \geq 0\}$ will be a pure-jump subordinator with infinite Lévy measure $v$, and $X_{\varepsilon}(\varepsilon \downarrow 0)$ will be the process consisting of those jumps of $X$ not exceeding $\varepsilon$ (its Lévy measure is thus the restriction of $v$ to $(0, \varepsilon])$. The expectation and variance of $X_{\varepsilon}(1)$ will be denoted by $\mu(\varepsilon)$ and $\sigma^{2}(\varepsilon)$, respectively. Explicitly, $\mu(\varepsilon)=\int_{(0, \varepsilon]} x \nu(\mathrm{d} x)$ and $\sigma^{2}(\varepsilon)=\int_{(0, \varepsilon]} x^{2} v(\mathrm{~d} x)$ (cf., e.g. [14, p. 163]). It is natural to look for a weak limit of $X_{\varepsilon} / \mu(\varepsilon)$. This is obviously important from the point of view of approximating the small jump part, $X_{\varepsilon}$, which is often needed. The reason why we consider only the $v((0, \infty))=\infty$ case is very simple: for pure-jump compound Poisson subordinators, the limit, assuming that $\mu(\varepsilon)>0$ for all $\varepsilon>0$, is trivially the zero process.

We will write $\Pi_{[\psi(\varepsilon)]}$ to denote the Lévy measure of $X_{\varepsilon} / \psi(\varepsilon)$, where $\psi(\varepsilon)=\mu(\varepsilon)$ or $\psi(\varepsilon)=\varepsilon$, unless otherwise indicated. To be more precise, the argument of $\psi$ can be extended

Received 24 June 2008; revision received 19 May 2009.

* Postal address: Department of Mathematics, Bar Ilan University, 52900 Ramat-Gan, Israel.

Email address: green355@netvision.net.il

A preprint version of this paper forms part of the author's $\mathrm{PhD}$ thesis, prepared at Bar Ilan University under the supervision of Prof. E. Merzbach. Work supported by the Doctoral Fellowship of Excellence, Bar Ilan University. 
to include $\varepsilon_{n}, \tilde{\varepsilon}_{n}$, etc. Thus, for example, $\Pi_{\left[\tilde{\varepsilon}_{k}\right]}$ is the Lévy measure of $X_{\tilde{\varepsilon}_{k}} / \tilde{\varepsilon}_{k}$, even if $\tilde{\varepsilon}_{k}$ is defined equal to $\varepsilon / 2^{k-1}$. Obviously, and importantly, $\Pi_{[\psi(\varepsilon)]}$ is concentrated on $(0, \varepsilon / \psi(\varepsilon)]$. By ' $\Rightarrow$ ' we denote weak convergence in the space $D[0, \infty)$ of càdlàg functions endowed with the Skorokhod topology (for details, we refer the reader to [9] and reference 198 therein), and by ' $\stackrel{\mathrm{D}}{\rightarrow}$ ' we denote convergence in distribution of random variables (RVs). Of particular importance in this paper is a pure-jump subordinator that we denote by $X_{\star}^{c}$, characterized by a Lévy measure $\Pi_{\star}^{c}$ given by

$$
\Pi_{\star}^{c}(\mathrm{~d} x)=\mathbf{1}_{(0,1]}(x) \frac{c}{x} \mathrm{~d} x, \quad c>0 \text { fixed } .
$$

It follows that, for any $a>0, a X_{\star}^{c}$ has Lévy measure $\mathbf{1}_{(0, a]}(x) c x^{-1} \mathrm{~d} x$ (cf. (1.2), below). We further denote by $\mathfrak{X}_{\star}^{c}$ the class of pure-jump subordinators $X$ such that $X_{\varepsilon} / \varepsilon \Rightarrow X_{\star}^{c}$; if we wish to leave $c$ unspecified, we denote the class by $\mathfrak{X}_{\star}$. The most important example of a process in $\mathfrak{X}_{\star}$ is the gamma process, as follows immediately from Corollary 2.3. This process has Lévy density $\rho(x)=\mathbf{1}_{(0, \infty)}(x) c \mathrm{e}^{-\lambda x} x^{-1}(c, \lambda>0)$, and will be denoted $\Gamma_{c, \lambda}$. Accordingly, $X(t) \sim \operatorname{gamma}(c t, \lambda), t>0$, meaning that $X(t)$ has density function $f(x ; t)=$ $\lambda^{c t} x^{c t-1} \mathrm{e}^{-\lambda x} / \Gamma(c t), x>0$.

We recall that a sequence of Lévy processes cannot converge to anything but a Lévy process (the stationary, independent increments property holds in the limit due to convergence of characteristic functions). In particular, a sequence of subordinators cannot converge to anything but a subordinator, since subordinators are the only Lévy processes with marginal distributions supported on $\mathbb{R}_{+}$. Moreover, it is well known that the weak convergence of Lévy processes reduces to the weak convergence of the marginal distributions at $t=1$ (see, e.g. [9, Corollary VII.3.6]). Thus, $X_{\varepsilon} / \psi(\varepsilon)$ converges weakly to some subordinator $Y$ if and only if $X_{\varepsilon}(1) / \psi(\varepsilon) \stackrel{\text { D }}{\rightarrow} Y(1)$.

For a subordinator $Y$ with drift $\beta \geq 0$ and Lévy measure $Q$, the Laplace transform of $Y(t)$ is given by (see, e.g. Equation (21.1) of [14])

$$
\mathrm{E}\left[\mathrm{e}^{-u Y(t)}\right]=\exp \left[t\left(-\beta u+\int_{(0, \infty)}\left(\mathrm{e}^{-u x}-1\right) Q(\mathrm{~d} x)\right)\right], \quad u \geq 0 .
$$

In accordance with [10, Chapter 15], we say that $Y$ is a subordinator with characteristics $(\beta, Q)$. A very useful tool for determining convergence of infinitely divisible distributions on $\mathbb{R}_{+}$ (i.e. marginal distributions of subordinators) is Theorem 15.14(ii) of [10]. This theorem, adapted to our setting, yields the following statement. Let $Y$ be a subordinator with characteristics $(\beta, Q)$, and fix any $h>0$ with $Q(\{h\})=0$. Then $X_{\varepsilon}(1) / \psi(\varepsilon) \stackrel{\text { D }}{\rightarrow} Y(1)$ if and only if the following two conditions hold:

(i) $\lim _{\varepsilon \downarrow 0} \int_{(0, h]} x \Pi_{[\psi(\varepsilon)]}(\mathrm{d} x)=\beta+\int_{(0, h]} x Q(\mathrm{~d} x)$,

(ii) $\lim _{\varepsilon \downarrow 0} \Pi_{[\psi(\varepsilon)]}((x, \varepsilon / \psi(\varepsilon)])=Q((x, \infty))$ for all $x>0$ with $Q(\{x\})=0$.

Condition (ii) can be denoted as $\Pi_{[\psi(\varepsilon)]} \stackrel{\mathrm{v}}{\rightarrow} Q$ (read ' $\Pi_{[\psi(\varepsilon)]}$ converges vaguely to $Q$ ').

Let $\mathcal{B}((0, \varepsilon / \psi(\varepsilon)])$ denote the Borel subsets of $(0, \varepsilon / \psi(\varepsilon)]$. By identifying the jumps of $X_{\varepsilon} / \psi(\varepsilon)$ whose size lies in $B \in \mathscr{B}((0, \varepsilon / \psi(\varepsilon)])$ with those of $X$ whose size lies in $\psi(\varepsilon) B$ $(=\{\psi(\varepsilon) x: x \in B\})$, we find that the measures $\Pi_{[\psi(\varepsilon)]}$ and $v$ are related by

$$
\Pi_{[\psi(\varepsilon)]}(B)=v(\psi(\varepsilon) B) \quad \text { for all } B \in \mathscr{B}((0, \varepsilon / \psi(\varepsilon)]) .
$$


Equivalently to (1.3), for any nonnegative measurable function $f$, it holds that

$$
\int_{B} f(x) \Pi_{[\psi(\varepsilon)]}(\mathrm{d} x)=\int_{\psi(\varepsilon) B} f\left(\frac{x}{\psi(\varepsilon)}\right) \nu(\mathrm{d} x) \text { for all } B \in \mathcal{B}((0, \varepsilon / \psi(\varepsilon)]) .
$$

Remark 1.1. The continuity of the parameter $\varepsilon$ is very significant. We have $X_{\varepsilon} / \psi(\varepsilon) \Rightarrow Y$ as $\varepsilon \downarrow 0$ if and only if $X_{\varepsilon_{n}} / \psi\left(\varepsilon_{n}\right) \Rightarrow Y$ for any sequence $\varepsilon_{n} \downarrow 0$. We shall see later on that the limit as $\varepsilon \downarrow 0$ of $\mu(\varepsilon) / \varepsilon$, if it exists in $[0, \infty]$, determines the weak limit of $X_{\varepsilon} / \mu(\varepsilon)$ as $\varepsilon \downarrow 0$. But, if $\mu(\varepsilon) / \varepsilon$ does not converge to a limit in $[0, \infty]$ then different choices of sequences $\varepsilon_{n} \downarrow 0$ may lead to different weak limits of $X_{\varepsilon_{n}} / \mu\left(\varepsilon_{n}\right)$ (see, e.g. Examples 3.8 and 3.9). This point is nicely illustrated in [2, Section 4$]$ in the context of approximating small jumps of arbitrary Lévy processes.

We now introduce the generalized Dickman distribution. Adapting the terminology and notation of [13], we shall say that a RV $Z$ has a generalized Dickman distribution with shape parameter $\theta>0$, denoted by $Z \sim \operatorname{GD}(\theta)$, if its Laplace transform is given by (see [13, Proposition 3])

$$
\mathrm{E}\left[\mathrm{e}^{-u Z}\right]=\exp \left[\theta \int_{0}^{1} \frac{\mathrm{e}^{-u x}-1}{x} \mathrm{~d} x\right], \quad u \geq 0 .
$$

The RV $Z$ is commonly represented as [13, Equation (8)]

$$
Z=U_{1}^{1 / \theta}+\left(U_{1} U_{2}\right)^{1 / \theta}+\left(U_{1} U_{2} U_{3}\right)^{1 / \theta}+\cdots
$$

where the $U_{i}$ are independent and identically distributed (i.i.d.) uniform( $(0,1) \mathrm{RVs}$. From (1.5), it follows that the one-dimensional distributions of the process $X_{\star}^{c}$ are GD: $X_{\star}^{c}(t) \sim \mathrm{GD}(c t)$, $t>0$.

Suppose that $\mu(\varepsilon) / \varepsilon \rightarrow c$ as $\varepsilon \downarrow 0(0<c<\infty)$. Then, by our Theorem $2.1, X_{\varepsilon} / \mu(\varepsilon) \Rightarrow$ $c^{-1} X_{\star}^{c}$ as $\varepsilon \downarrow 0$. More elegantly and conveniently, $X_{\varepsilon} / \varepsilon \Rightarrow X_{\star}^{c}$. A thorough characterization of the class $\mathfrak{X}_{\star}^{c}$, in terms of the Lévy measure $v$ of $X$, is provided in Proposition 2.1. The attractiveness of the approximation $X_{\varepsilon} \cong \varepsilon X_{\star}^{c}$, the one valid for processes $X \in \mathfrak{X}_{\star}^{c}$, is due to the facts described in the next four paragraphs.

The GD distribution (and, in particular, its probability density and distribution functions) has been extensively studied in the literature. It occurs, among others, in number-theoretical [7], [18] and combinatorial [1] contexts. It also appears in connection with the PoissonDirichlet distribution [6], [8]. Many of the basic properties of the GD distribution can be found in [1, Section 4.2], [6], [7], [13], [15], [17, pp. 90-95], and [18]. (Of particular importance is the $\mathrm{GD}(1)$ distribution, whose density is a normalization of the celebrated Dickman function.) Two simple examples of sequences $Z_{n}$ of RVs that converge in distribution to $Z \sim \mathrm{GD}(\theta)$ are: $Z_{n}=\sum_{i=1}^{n} \exp \left[-(n / \theta) U_{i}\right]$ where the $U_{i}$ are i.i.d. uniform $(0,1)[18$, p. 501] and $Z_{n}=n^{-1} \sum_{i=1}^{n} i W_{i}$, where the $W_{i}$ are independent Poisson with means $\mathrm{E}\left[W_{i}\right]=$ $\theta / i$ [1, Theorem 4.6]. Some recent results where the GD distribution arises as the limit in distribution of certain sequences of RVs are Theorem 1 of [13] and the theorems in [11]. Our example of the class $\mathfrak{X}_{\star}$ joins the growing list of instances where the GD distribution is encountered as a limit law (here, in an infinite-dimensional sense). The extensive collection of results on this distribution is potentially of great use with regard to the class $\mathfrak{X}_{\star}$; in fact, results in [18] and [7] on the maximum and asymptotic behavior of the GD density function are fundamental in the proof of the asymptotic formula (4.17) in our Theorem 4.2. 
The process $X_{\star}^{c}$ admits the following series representation in the time interval $[0, T]$ :

$$
X_{\star}^{c}(t)=\sum_{j=1}^{\infty}\left(U_{1} \cdots U_{j}\right)^{1 /(c T)} \mathbf{1}_{\left\{\hat{U}_{j} \leq t\right\}}
$$

(see [4]), where $\left\{U_{j}\right\}$ and $\left\{\hat{U}_{j}\right\}$ are independent sequences of i.i.d. uniform $(0,1)$ and i.i.d. uniform $(0, T) \mathrm{RVs}$, respectively. Thus, $X_{\star}^{c}$ can be easily simulated on $[0, T]$, with high accuracy and short processing time if $c T$ is not too large. The fact that the $\mathrm{RV} X_{\star}^{c}(t)$ is easy to simulate can be of great use in evaluating probabilities of interest using Monte Carlo simulations (in this context, see the definition of $b_{c, \alpha}(x, t)$ in Theorem 4.2, and the paragraph relating to Table 1 in Section 4). It is important to note here that the distribution function (DF) of $X_{\star}^{c}(t)$ is not easily calculated by analytical or partially analytical methods for values of $x$ far from the origin (see the end of Section 4).

The class $\mathfrak{X}_{\star}$ is rich and contains several interesting examples. The most prominent examples are the $\Gamma_{c, \lambda}$ process and the process $a X_{\star}^{c}(a>0)$. As follows trivially from our Corollary 2.3 (and has already been shown in [4]), any Lévy density $\rho$ satisfying $\rho(x) \sim c / x$ as $x \downarrow 0$ corresponds to a process $X \in \mathfrak{X}_{\star}^{c}(f \sim g$ means $f / g \rightarrow 1)$. It follows that $\mathfrak{X}_{\star}^{c}$ contains a fundamental class of self-decomposable subordinators; see Example 3.1. Interesting examples of processes $X \in \mathfrak{X}_{\star}^{c}$ with discrete Lévy measure are easily obtained using Proposition 2.2. Moreover, if $Z$ is a Lévy process of the form $Z=X_{1}-X_{2}$, where $X_{1} \in \mathfrak{X}_{\star}^{c_{1}}$ and $X_{2} \in \mathfrak{X}_{\star}^{c_{2}}$ are independent, and if $Z_{\varepsilon}$ consists of those jumps of $Z$ not exceeding $\varepsilon$ in absolute value, then one is led to the approximation $Z_{\varepsilon} \cong \varepsilon\left[X_{\star}^{c_{1}}-X_{\star}^{c_{2}}\right]$ with $X_{\star}^{c_{1}}$ and $X_{\star}^{c_{2}}$ independent; indeed, $Z_{\varepsilon} / \varepsilon \Rightarrow X_{\star}^{c_{1}}-X_{\star}^{c_{2}}$. The primary example of such a process $Z$ is the variance gamma process; see Example 3.12.

We shall see in Theorem 2.1 that if $\mu(\varepsilon) / \varepsilon$ converges to 0 or $\infty$ as $\varepsilon \downarrow 0$, then $X_{\varepsilon} / \mu(\varepsilon)$ converges weakly as $\varepsilon \downarrow 0$ to the zero process or the unit drift process $t$, respectively. Moreover, by the same theorem, the weak convergence of $X_{\varepsilon} / \mu(\varepsilon)$ implies the convergence of $\mu(\varepsilon) / \varepsilon$ to a limit in $[0, \infty]$ in the case where $v$ is continuous near the origin. These facts, taking into account the $\mu(\varepsilon) / \varepsilon \rightarrow c \in(0, \infty)$ case already considered, underline the basic importance of the process $X_{\star}^{c}$ in our context.

Remark 1.2. As described later on in this section and in Example 3.12, approximations of small jumps of gamma and variance gamma processes, similar to the ones presented in the present paper, have already been identified in the literature. The benefits of our approach will be evident.

As stated in the abstract, this paper completes, in some respects, the study in [2]. There, the authors considered approximations of small jumps of Lévy processes by a Brownian motion with small variance and drift. For simplicity and to highlight the relationship to the present paper, we suppose that the process is a pure-jump subordinator with infinite Lévy measure (the general case is very similar). Combining Theorem 2.1 and Proposition 2.1 of [2], we infer the following statement. Let $W$ be a standard Brownian motion, and let $A_{\varepsilon}$ be the drift process defined by $A_{\varepsilon}(t)=\mu(\varepsilon) t$. Then

$$
\frac{X_{\varepsilon}-A_{\varepsilon}}{\sigma(\varepsilon)} \Rightarrow W \quad \text { as } \varepsilon \downarrow 0
$$

if and only if, for each $\kappa>0$,

$$
\sigma(\kappa \sigma(\varepsilon) \wedge \varepsilon) \sim \sigma(\varepsilon) \quad \text { as } \varepsilon \downarrow 0 .
$$


Condition (1.8) is implied by

$$
\lim _{\varepsilon \downarrow 0} \frac{\sigma(\varepsilon)}{\varepsilon}=\infty .
$$

Moreover, if $v$ is continuous near the origin then (1.8) and (1.9) are equivalent.

Remark 1.3. Even if $\liminf _{\varepsilon \downarrow 0}(\mu(\varepsilon) / \varepsilon)=0$, which implies that $\liminf _{\varepsilon \downarrow 0}(\sigma(\varepsilon) / \varepsilon)=0$ by $\sigma^{2}(\varepsilon) / \varepsilon^{2} \leq \mu(\varepsilon) / \varepsilon$, then (1.8), and, hence, (1.7) also, can still hold. See Example 3.7.

Now we are ready to show the relationship to our work. The weak convergence in (1.7), if it holds, leads to the following approximation (useful for simulation purposes):

$$
X(t) \cong \mu(\varepsilon) t+\sigma(\varepsilon) W(t)+X^{\varepsilon}(t), \quad t \geq 0,
$$

where $X^{\varepsilon}:=X-X_{\varepsilon}$ (a compound Poisson process with Lévy measure $v_{\mid\{x>\varepsilon\}}$ ) is independent of $W$. In Proposition 2.3 we show that (1.7) implies that $\sigma(\varepsilon) / \mu(\varepsilon) \rightarrow 0$ and, in turn, that $X_{\varepsilon} / \mu(\varepsilon)$ converges weakly to the unit drift process $t$. Thus, even if the normal approximation for $X_{\varepsilon}$ is valid, we can neglect the Brownian term $\sigma(\varepsilon) W(t)$ in (1.10) and still get satisfactory approximations/simulations. As an example, for the strictly $\alpha$-stable subordinator $(0<\alpha<1)$ in Example 3.1, it holds that $\sigma(\varepsilon) / \mu(\varepsilon)=(1-\alpha) \varepsilon^{\alpha / 2} / \sqrt{(2-\alpha) b}(b>0)$. Here we note that the weak convergence of $X_{\varepsilon} / \mu(\varepsilon)$ to the unit drift process does not necessarily imply (1.7). See Example 3.11. The above facts only add importance to the question of weak convergence of $X_{\varepsilon} / \mu(\varepsilon)$.

Remark 1.4. Conforming to [2], we should have defined $X_{\varepsilon}$ to be the sum of jumps whose sizes are strictly less than $\varepsilon$, and, accordingly, $\mu(\varepsilon)=\int_{(0, \varepsilon)} x \nu(\mathrm{d} x)$ and $\sigma^{2}(\varepsilon)=\int_{(0, \varepsilon)} x^{2} \nu(\mathrm{d} x)$. However, it can be easily realized that this distinction plays no role here. (Of course, this distinction is irrelevant for Lévy measures which are continuous near the origin.) The guiding principle behind this fact is that, for any $\varepsilon>0$, arbitrarily small but fixed, $(0, \varepsilon]=\bigcap_{\delta>0}(0, \varepsilon+\delta]$ and $(0, \varepsilon)=\bigcup_{\delta>0}(0, \varepsilon-\delta]$.

As Corollary 2.5 states (and follows readily from above), the normal approximation of small jumps is not valid (that is, (1.7) does not hold) for processes in $\mathfrak{X}_{\star}$. It has already been pointed out [5] that in the case where $X$ is a gamma process (1.7) does hold if we replace $W$ by some other (Lévy) process. Let $X$ be a $\Gamma_{c, \lambda}$ process. By Example 5.2(a) of [5], adapted to our notation,

$$
\frac{X_{\varepsilon}-A_{\varepsilon}}{\sigma(\varepsilon)} \Rightarrow Y^{c} \quad \text { as } \varepsilon \downarrow 0,
$$

where $Y^{c}$ admits the Lévy-Khintchine representation (see, e.g. [14])

$$
\mathrm{E}\left[\exp \left[\mathrm{i} u Y^{c}(1)\right]\right]=\exp \left[\mathrm{i} a u+\int_{\mathbb{R}}\left(\mathrm{e}^{\mathrm{i} u x}-1-\mathrm{i} u x \mathbf{1}_{[-1,1]}(x)\right) \pi(\mathrm{d} x)\right], \quad u \in \mathbb{R},
$$

with $a=c(1-\sqrt{2 / c}) \wedge 0$ and $\pi(\mathrm{d} x)=\mathbf{1}_{(0, \sqrt{2 / c]}}(x) c x^{-1} \mathrm{~d} x$. (The proof was based on Theorem 15.14(i) of [10]—a commonly used result for showing convergence of infinitely divisible distributions on $\mathbb{R}$.) From this, using the fact that $\pi(\mathrm{d} x)$ is the Lévy measure of $(2 / c)^{1 / 2} X_{\star}^{c}$, we can find $Y^{c}(t) \stackrel{\mathrm{D}}{=}-(2 c)^{1 / 2} t+(2 / c)^{1 / 2} X_{\star}^{c}(t)$. The connection with our result, $X_{\varepsilon} / \varepsilon \Rightarrow X_{\star}^{c}$, is then established by noting that $\sigma(\varepsilon) \sim(c / 2)^{1 / 2} \varepsilon$ and $A_{\varepsilon}(t) / \sigma(\varepsilon) \rightarrow(2 c)^{1 / 2} t$ as $\varepsilon \downarrow 0$. It is worth noting that in simple cases it may be preferable to use Lévy's continuity theorem rather than Theorem 15.14 (part (i) or (ii)) of [10] to show weak convergence of Lévy processes (on $\mathbb{R}$ or $\mathbb{R}_{+}$, respectively). For example, with $X$ as above, we obtain the desired 
convergence $X_{\varepsilon} / \varepsilon \Rightarrow X_{\star}^{c}$ as $\varepsilon \downarrow 0$ (and, hence, also (1.11)) using the continuity theorem for Laplace transforms applied to

$$
\mathrm{E}\left[\exp \left[-u\left(\frac{X_{\varepsilon}(1)}{\varepsilon}\right)\right]\right]=\exp \left[\int_{0}^{1}\left(\mathrm{e}^{-u x}-1\right) \frac{c \mathrm{e}^{-\lambda \varepsilon x}}{x} \mathrm{~d} x\right], \quad u \geq 0 .
$$

However, as we would expect, the heavier tool of [10] (Theorem 15.14(ii) for the purposes of this paper) is, in general, the more appropriate one to use.

The rest of the paper is organized as follows. In Section 2 we state and prove the general results of this paper. The main results are Theorem 2.1 and Proposition 2.1. Both have already been mentioned above, the second as characterizing the class $\mathfrak{X}_{\star}^{c}$. In this section we also derive the asymptotics of $v((\varepsilon, \infty))$ and $\mathrm{P}(X(t) \leq \varepsilon)$ as $\varepsilon \downarrow 0$ for processes $X \in \mathfrak{X}_{\star}^{c}$ (Proposition 2.4 and Theorem 2.2, respectively). Section 3 is devoted to examples related to Sections 1 and 2. In Section 4 we concentrate on the class of pure-jump subordinators $X$ with Lévy density $\rho$ such that $c-x \rho(x)=x^{\alpha} L(x)$ for some constant $\alpha>0$ and function $L$ slowly varying at 0 . We recall the definition of slow variation (at 0 ) in that section. This class, which is contained in $\mathfrak{X}_{\star}^{c}$, contains some significant class of self-decomposable subordinators, in particular the $\Gamma_{c, \lambda}$ process. These get extra focus. We analyze the difference between the DFs of $X(t)$ and its approximation formed by replacing $X_{\varepsilon}(t)$ by $\varepsilon X_{\star}^{c}(t)$, and the difference between the DFs of $X_{\varepsilon}(t) / \varepsilon$ and $X_{\star}^{c}(t)$. A brief account of the calculation of the GD probability distribution and density functions concludes the paper.

\section{General results}

With the preliminaries established at the beginning of Section 1, we now state and prove our first main result. Corollary 2.1, below, then follows immediately.

Theorem 2.1. The following statements hold, as $\varepsilon \downarrow 0$.

(a) If $\mu(\varepsilon) / \varepsilon \rightarrow c$, where $0<c<\infty$, then $X_{\varepsilon} / \mu(\varepsilon)$ converges weakly to $c^{-1} X_{\star}^{c}$.

(b) If $\mu(\varepsilon) / \varepsilon \rightarrow 0$ then $X_{\varepsilon} / \mu(\varepsilon)$ converges weakly to the zero process.

(c) If $\mu(\varepsilon) / \varepsilon \rightarrow \infty$ then $X_{\varepsilon} / \mu(\varepsilon)$ converges weakly to the unit drift process.

Finally, suppose that none of the above conditions on $\mu(\varepsilon) / \varepsilon$ is satisfied. Then the following statement holds.

(d) If $v$ is continuous near the origin then $X_{\varepsilon} / \mu(\varepsilon)$ necessarily fails to converge. Otherwise, $X_{\varepsilon} / \mu(\varepsilon)$ may or may not converge. Moreover, if $\lim \sup _{\varepsilon \downarrow 0}(\mu(\varepsilon) / \varepsilon)=\infty$ then the only possible limit is the unit drift process.

Proof. We divide the proof into steps A-D, corresponding to statements (a)-(d) above.

Step $A$. We assume that $\mu(\varepsilon) / \varepsilon \rightarrow c, c>0$ real. It suffices to prove that $X_{\varepsilon} / \varepsilon \Rightarrow X_{\star}^{c}$. Now, $X_{\star}^{c}$ has characteristics $\left(0, \Pi_{\star}^{c}\right)$; so substituting from definition (1.1) and applying conditions (i) and (ii) from Section 1, the latter convergence holds if and only if

$$
\lim _{\varepsilon \downarrow 0} \int_{(0,1]} x \Pi_{[\varepsilon]}(\mathrm{d} x)=c
$$

and, for all $0<x<1$,

$$
\lim _{\varepsilon \downarrow 0} \Pi_{[\varepsilon]}((x, 1])=c \log \left(\frac{1}{x}\right) .
$$


Since $\int_{(0,1]} x \Pi_{[\varepsilon]}(\mathrm{d} x)=\mathrm{E}\left[X_{\varepsilon}(1) / \varepsilon\right]=\mu(\varepsilon) / \varepsilon$, condition (2.1) is satisfied. To prove the second condition, let us first define (finite) measures $\eta_{\varepsilon}$ and $\eta$ on $(0,1]$ by $\eta_{\varepsilon}(\mathrm{d} x)=x \Pi_{[\varepsilon]}(\mathrm{d} x)$ and $\eta(\mathrm{d} x)=\mathbf{1}_{(0,1]}(x) c \mathrm{~d} x$. Fix any $0<x \leq 1$. Then, by (1.4) and $\mu(\varepsilon) / \varepsilon \rightarrow c$,

$$
\eta_{\varepsilon}((0, x])=\int_{(0, x]} u \Pi_{[\varepsilon]}(\mathrm{d} u)=\frac{x}{\varepsilon x} \int_{(0, \varepsilon x]} u v(\mathrm{~d} u) \rightarrow c x=\eta((0, x]),
$$

from which it follows that $\int_{(x, 1]} u^{-1} \eta_{\varepsilon}(\mathrm{d} u) \rightarrow \int_{(x, 1]} u^{-1} \eta(\mathrm{d} u)$. Therefore, $\int_{(x, 1]} \Pi_{[\varepsilon]}(\mathrm{d} u) \rightarrow$ $\int_{x}^{1}(c / u) \mathrm{d} u$, and condition (2.2) is established.

Step B. We assume that $\mu(\varepsilon) / \varepsilon \rightarrow 0$. Analogously to conditions (2.1) and (2.2) above, $X_{\varepsilon} / \mu(\varepsilon)$ converges weakly to the zero process if and only if

$$
\lim _{\varepsilon \downarrow 0} \int_{(0,1]} x \Pi_{[\mu(\varepsilon)]}(\mathrm{d} x)=0
$$

and, for all $x>0$,

$$
\lim _{\varepsilon \downarrow 0} \Pi_{[\mu(\varepsilon)]}\left(\left(x, \frac{\varepsilon}{\mu(\varepsilon)}\right]\right)=0 .
$$

A straightforward application of (1.4) yields $\int_{(0,1]} x \Pi_{[\mu(\varepsilon)]}(\mathrm{d} x)=\mu(\varepsilon)^{-1} \int_{(0, \mu(\varepsilon)]} x v(\mathrm{~d} x)$ for $\varepsilon / \mu(\varepsilon) \geq 1$. Since the right-hand side is $\mu(\mu(\varepsilon)) / \mu(\varepsilon)$, condition (2.3) is satisfied. The second condition, (2.4), is proved as follows. Fix $x>0$. If $\varepsilon / \mu(\varepsilon)>M$, we have, using (1.4),

$$
\begin{aligned}
\Pi_{[\mu(\varepsilon)]}\left(\left(x, \frac{\varepsilon}{\mu(\varepsilon)}\right]\right) & =\Pi_{[\mu(\varepsilon)]}((x, M])+\Pi_{[\mu(\varepsilon)]}\left(\left(M, \frac{\varepsilon}{\mu(\varepsilon)}\right]\right) \\
& \leq \frac{M}{x M} \int_{(x, M]} u \Pi_{[\mu(\varepsilon)]}(\mathrm{d} u)+\frac{1}{M} \int_{(M, \varepsilon / \mu(\varepsilon)]} u \Pi_{[\mu(\varepsilon)]}(\mathrm{d} u) \\
& =\frac{M}{x} \frac{1}{\mu(\varepsilon) M} \int_{(\mu(\varepsilon) x, \mu(\varepsilon) M]} u v(\mathrm{~d} u)+\frac{1}{M} \frac{1}{\mu(\varepsilon)} \int_{(\mu(\varepsilon) M, \varepsilon]} u v(\mathrm{~d} u) \\
& \leq \frac{M}{x} \frac{\mu(\mu(\varepsilon) M)}{\mu(\varepsilon) M}+\frac{1}{M} .
\end{aligned}
$$

So clearly condition (2.4) is satisfied.

Step $C$. We assume that $\mu(\varepsilon) / \varepsilon \rightarrow \infty$. The unit drift process has characteristics $(1, \mathbf{0})$, where $\mathbf{0}$ denotes the zero measure on $(0, \infty)$. Since $\varepsilon / \mu(\varepsilon) \rightarrow 0, \Pi_{[\mu(\varepsilon)]} \stackrel{\mathrm{v}}{\rightarrow} \mathbf{0}$. Hence, statement (c) will follow once we show that

$$
\lim _{\varepsilon \downarrow 0} \int_{(0, \varepsilon / \mu(\varepsilon)]} x \Pi_{[\mu(\varepsilon)]}(\mathrm{d} x)=1 .
$$

Indeed, $\int_{(0, \varepsilon / \mu(\varepsilon)]} x \Pi_{[\mu(\varepsilon)]}(\mathrm{d} x)=\mathrm{E}\left[X_{\varepsilon}(1) / \mu(\varepsilon)\right]=1$. (Alternatively, it is easy to prove that $X_{\varepsilon}(1) / \mu(\varepsilon) \stackrel{\mathrm{D}}{\rightarrow} 1$ using Chebyshev's inequality, noting that $\operatorname{var}\left[X_{\varepsilon}(1) / \mu(\varepsilon)\right] \rightarrow 0$.)

Step $D$. We assume that $\mu(\varepsilon) / \varepsilon$ does not meet any of the previous conditions. Let us moreover assume that $v$ is continuous near 0 . Hence, the monotonically increasing function $\mu(\varepsilon), \varepsilon>0$, is also continuous near 0 , with $\mu(0+)=0$. We first claim that if $\lim \sup _{\varepsilon \downarrow 0}(\mu(\varepsilon) / \varepsilon)<\infty$ then $X_{\varepsilon} / \mu(\varepsilon)$ fails to converge. Suppose, by contradiction, that $X_{\varepsilon} / \mu(\varepsilon) \Rightarrow Y$ for a subordinator $Y$ with characteristics $(\beta, Q)$, and fix $h>0$ with $Q(\{h\})=0$ and $h<\liminf _{\varepsilon \downarrow 0}(\varepsilon / \mu(\varepsilon))$. Applying condition (i) from Section 1, we obtain

$$
\lim _{\varepsilon \downarrow 0} \frac{\mu(h \mu(\varepsilon))}{h \mu(\varepsilon)}=\lim _{\varepsilon \downarrow 0} \frac{1}{h \mu(\varepsilon)} \int_{(0, h \mu(\varepsilon)]} x v(\mathrm{~d} x)=\frac{1}{h} \lim _{\varepsilon \downarrow 0} \int_{(0, h]} x \Pi_{[\mu(\varepsilon)]}(\mathrm{d} x)=\alpha_{h},
$$


where $\alpha_{h}=\left(\beta+\int_{(0, h]} x \underset{y}{ }(\mathrm{~d} x)\right) / h$. For any $y$ in $\left(\liminf _{\varepsilon \downarrow 0}(\mu(\varepsilon) / \varepsilon), \lim \sup _{\varepsilon \downarrow 0}(\mu(\varepsilon) / \varepsilon)\right)$, there exists a sequence $\varepsilon_{n}^{y} \downarrow 0$ with $\mu\left(\varepsilon_{n}^{y}\right) / \varepsilon_{n}^{y}=y$ for all $n$ and a corresponding sequence $\tilde{\varepsilon}_{n}^{y} \downarrow 0$ with $h \mu\left(\tilde{\varepsilon}_{n}^{y}\right)=\varepsilon_{n}^{y}$ for all $n$. Substituting into (2.5) gives $y=\alpha_{h}$, a contradiction.

We now claim (under the starting assumptions) that $X_{\varepsilon} / \mu(\varepsilon)$ also fails to converge if $\lim \sup _{\varepsilon \downarrow 0}(\mu(\varepsilon) / \varepsilon)=\infty$. Assuming the latter, there exists a sequence $\varepsilon_{n} \downarrow 0$ with $\mu\left(\varepsilon_{n}\right) / \varepsilon_{n} \rightarrow$ $\infty$. Then, as in step C, $X_{\varepsilon_{n}} / \mu\left(\varepsilon_{n}\right)$ converges weakly to the unit drift process, which is therefore the only possible limit of $X_{\varepsilon} / \mu(\varepsilon)$. Suppose, by contradiction, that it is the limit. Fix any $y$ in $\left.\liminf _{\varepsilon \downarrow 0}(\mu(\varepsilon) / \varepsilon), \infty\right)$, and let $0<a<1 / y$. As before, there exists a sequence $\varepsilon_{n}^{y} \downarrow 0$ with $\mu\left(\varepsilon_{n}^{y}\right) / \varepsilon_{n}^{y}=y$ for all $n$ and a sequence $\tilde{\varepsilon}_{n} \downarrow 0$ with $a \mu\left(\tilde{\varepsilon}_{n}\right)=\varepsilon_{n}^{y}$ for all $n$. This time we take as the function $\psi(\varepsilon)$ in (1.4) the function $a \mu(\varepsilon)$. For any $n$ satisfying $\tilde{\varepsilon}_{n} /\left(a \mu\left(\tilde{\varepsilon}_{n}\right)\right) \geq 1$, we then have

$$
\int_{(0,1]} x \Pi_{\left[a \mu\left(\tilde{\varepsilon}_{n}\right)\right]}(\mathrm{d} x)=\frac{1}{a \mu\left(\tilde{\varepsilon}_{n}\right)} \int_{\left(0, a \mu\left(\tilde{\varepsilon}_{n}\right)\right]} x \nu(\mathrm{d} x)=\frac{\mu\left(a \mu\left(\tilde{\varepsilon}_{n}\right)\right)}{a \mu\left(\tilde{\varepsilon}_{n}\right)}=y<\frac{1}{a} .
$$

Since necessarily $X_{\tilde{\varepsilon}_{n}}(1) /\left(a \mu\left(\tilde{\varepsilon}_{n}\right)\right) \stackrel{\mathrm{D}}{\rightarrow} 1 / a$, we conclude from (2.6) that $a \mu\left(\tilde{\varepsilon}_{n}\right) / \tilde{\varepsilon}_{n}>1$ for all $n$ greater than some $n_{0}$. Hence, $\varepsilon_{n}^{y}>\tilde{\varepsilon}_{n}$ for all $n>n_{0}$ also. Let $n>n_{0}$. Then, using the monotonicity of $\mu$, we obtain $a \geq a \mu\left(\tilde{\varepsilon}_{n}\right) / \mu\left(\varepsilon_{n}^{y}\right)=\varepsilon_{n}^{y} / \mu\left(\varepsilon_{n}^{y}\right)=1 / y$, a contradiction.

In order to establish statement (d), we need to provide a pair of examples. We will do a little more. Examples 3.6 and 3.7, below, on the one hand and Examples 3.8 and 3.9, below, on the other hand, respectively show the convergence and the failing of convergence of $X_{\varepsilon} / \mu(\varepsilon)$, where in all the examples $v$ is discrete and $\mu(\varepsilon) / \varepsilon$ does not converge to a limit in $[0, \infty]$.

Corollary 2.1. Suppose that $v$ has a density $\rho$ near the origin. Then, as $\varepsilon \downarrow 0$,

(a) if $\lim _{x \downarrow 0} x \rho(x)=c$, where $0<c<\infty$, then $X_{\varepsilon} / \mu(\varepsilon)$ converges weakly to $c^{-1} X_{\star}^{c}$,

(b) if $\lim _{x \downarrow 0} x \rho(x)=0$ then $X_{\varepsilon} / \mu(\varepsilon)$ converges weakly to the zero process,

(c) if $\lim _{x \downarrow 0} x \rho(x)=\infty$ then $X_{\varepsilon} / \mu(\varepsilon)$ converges weakly to the unit drift process.

Here we note that $x \rho(x)$ need not converge to a limit in $[0, \infty]$ in order for one of the above convergences to hold (consider changing $\rho$ on a set of measure zero).

Remark 2.1. Clearly, $\mathrm{E}\left[X_{\varepsilon}(t) / \mu(\varepsilon)\right]=t$. Assume that $\mu(\varepsilon) / \varepsilon \rightarrow 0$. Then the interval $(0, \varepsilon / \mu(\varepsilon)]$, on which $\Pi_{[\mu(\varepsilon)]}$ is supported, spreads to infinity as $\varepsilon \downarrow 0$. Simultaneously, the measure itself gets thinner and thinner, and $X_{\varepsilon} / \mu(\varepsilon)$ converges weakly to the zero process. Let us show that the big jumps are those that keep $\mathrm{E}\left[X_{\varepsilon}(t) / \mu(\varepsilon)\right]$ constant. Fix any $M>0$ (large). If $\varepsilon / \mu(\varepsilon) \geq M$ then

$$
\int_{(0, M]} x \Pi_{[\mu(\varepsilon)]}(\mathrm{d} x)=\frac{M}{\mu(\varepsilon) M} \int_{(0, \mu(\varepsilon) M]} x v(\mathrm{~d} x)=M \frac{\mu(\mu(\varepsilon) M)}{\mu(\varepsilon) M} \rightarrow 0,
$$

from which our claim follows. As for the opposite case, $\mu(\varepsilon) / \varepsilon \rightarrow \infty$, the jump sizes tend to 0 as $\varepsilon \downarrow 0$, but their increased intensity keeps the expectation constant, and $X_{\varepsilon} / \mu(\varepsilon)$ converges weakly to the unit drift process.

The next corollary will be extended in our second main result, Proposition 2.1.

Corollary 2.2. The only possible limit laws of $X_{\varepsilon} / \varepsilon$, as $\varepsilon \downarrow 0$, are $X_{\star}^{c}$ (for any $c>0$ ) and the zero process. The first convergence holds if and only if $\mu(\varepsilon) / \varepsilon \rightarrow c$, while the second convergence holds if and only if $\mu(\varepsilon) / \varepsilon \rightarrow 0$. 
Proof. Suppose that $X_{\varepsilon} / \varepsilon \Rightarrow Y$ for some subordinator $Y$ with characteristics $(\beta, Q)$, and fix $0<h \leq 1$ with $Q(\{h\})=0$. Applying condition (i) from Section 1, we obtain

$$
\lim _{\varepsilon \downarrow 0} \frac{\mu(h \varepsilon)}{h \varepsilon}=\lim _{\varepsilon \downarrow 0} \frac{1}{h \varepsilon} \int_{(0, h \varepsilon]} x v(\mathrm{~d} x)=\frac{1}{h} \lim _{\varepsilon \downarrow 0} \int_{(0, h]} x \Pi_{[\varepsilon]}(\mathrm{d} x)=\alpha_{h},
$$

where $\alpha_{h}=\left(\beta+\int_{(0, h]} x Q(\mathrm{~d} x)\right) / h$. Thus, $\mu(\varepsilon) / \varepsilon$ tends to some nonnegative constant $\left(\alpha_{h}\right.$, independent of $h)$. Now rewrite $X_{\varepsilon} / \varepsilon$ as $(\mu(\varepsilon) / \varepsilon)\left(X_{\varepsilon} / \mu(\varepsilon)\right)$ and consider statements (a) and (b) of Theorem 2.1 to conclude the corollary.

The analogue of Corollary 2.1 for the process $X_{\varepsilon} / \varepsilon$ is straightforward. In particular, we have the following result.

Corollary 2.3. Suppose that $v$ has a density $\rho$ near the origin. If $\lim _{x \downarrow 0} x \rho(x)=c$, where $0<c<\infty$, then $X_{\varepsilon} / \varepsilon \Rightarrow X_{\star}^{c}$ as $\varepsilon \downarrow 0$.

As before, we note that the above condition is only a sufficient one.

We now further characterize the class $\mathfrak{X}_{\star}^{c}$ in terms of the Lévy measure of $X$.

Proposition 2.1. Each of the following conditions is necessary and sufficient for $X_{\varepsilon} / \varepsilon$ to converge weakly to $X_{\star}^{c}$ as $\varepsilon \downarrow 0$.

1. $\lim _{\varepsilon \downarrow 0}\left(1 / \varepsilon^{p}\right) \int_{(0, \varepsilon]} x^{p} v(\mathrm{~d} x)=c / p$ for all $p>0$.

2. $\lim _{\varepsilon \downarrow 0}\left(1 / \varepsilon^{p}\right) \int_{(0, \varepsilon]} x^{p} v(\mathrm{~d} x)=c / p$ for some $p>0$.

3. $\lim _{\varepsilon \downarrow 0} v((\varepsilon x, \varepsilon])=c \log (1 / x)$ for all $0<x<1$.

Proof. We have just seen that $X_{\varepsilon} / \varepsilon \Rightarrow X_{\star}^{c}$ if and only if $\mu(\varepsilon) / \varepsilon \rightarrow c$. Thus, in order to establish the proposition, it suffices to show that conditions 1,2 , and 3 are equivalent. We now show, in steps 1 and 2, that condition 2 implies condition 3, which in turn implies condition 1 (implies condition 2).

Step 1. By (1.3), condition 3 is equivalent to $\lim _{\varepsilon \downarrow 0} \Pi_{[\varepsilon]}((x, 1])=\int_{x}^{1}(c / u) \mathrm{d} u$ for all $0<x<1$-which can be rewritten as $\Pi_{[\varepsilon]} \stackrel{\mathrm{v}}{\rightarrow} \Pi_{\star}^{c}$. Now assume that $p$ satisfies condition 2 . We repeat the technique used in step A of Theorem 2.1, in a generalized form. Define (finite) measures $\eta_{\varepsilon}$ and $\eta$ on $(0,1]$ by $\eta_{\varepsilon}(\mathrm{d} x)=x^{p} \Pi_{[\varepsilon]}(\mathrm{d} x)$ and $\eta(\mathrm{d} x)=\mathbf{1}_{(0,1]}(x) x^{p-1} c \mathrm{~d} x$. Fix any $0<x \leq 1$. Then, by (1.4) and the assumption on $p$,

$$
\eta_{\varepsilon}((0, x])=\int_{(0, x]} u^{p} \Pi_{[\varepsilon]}(\mathrm{d} u)=\frac{x^{p}}{(\varepsilon x)^{p}} \int_{(0, \varepsilon x]} u^{p} v(\mathrm{~d} u) \rightarrow \frac{c x^{p}}{p}=\eta((0, x]),
$$

from which it follows that $\int_{(x, 1]} u^{-p} \eta_{\varepsilon}(\mathrm{d} u) \rightarrow \int_{(x, 1]} u^{-p} \eta(\mathrm{d} u)$. Hence, $\int_{(x, 1]} \Pi_{[\varepsilon]}(\mathrm{d} u) \rightarrow$ $\int_{x}^{1}(c / u) \mathrm{d} u$, and condition 3 is established. Thus, condition 2 implies condition 3 .

Step 2. Now assume that condition 3 holds, and fix any $p>0$. Define $\tilde{\varepsilon}_{k}=\varepsilon / 2^{k-1}$, and apply (1.4) to obtain, for every $N=1,2, \ldots$,

$$
\frac{1}{\varepsilon^{p}} \int_{\left(\varepsilon / 2^{N}, \varepsilon\right]} x^{p} \nu(\mathrm{d} x)=\frac{1}{\varepsilon^{p}} \sum_{k=1}^{N} \int_{\left(\tilde{\varepsilon}_{k} / 2, \tilde{\varepsilon}_{k}\right]} x^{p} \nu(\mathrm{d} x)=\sum_{k=1}^{N} \frac{1}{2^{(k-1) p}} \int_{(1 / 2,1]} x^{p} \Pi_{\left[\tilde{\varepsilon}_{k}\right]}(\mathrm{d} x) .
$$

Since condition 3 is equivalent to $\Pi_{[\varepsilon]} \stackrel{\mathrm{v}}{\rightarrow} \Pi_{\star}^{c}$, it follows that

$$
\int_{(1 / 2,1]} x^{p} \Pi_{[\varepsilon]}(\mathrm{d} x) \rightarrow \int_{1 / 2}^{1} x^{p}\left(\frac{c}{x}\right) \mathrm{d} x=\left(\frac{c}{p}\right)\left(1-2^{-p}\right) .
$$


Using the triangle inequality and noting that $\tilde{\varepsilon}_{k} \leq \varepsilon$, we conclude that, for every $\eta>0$, there exists a $\delta>0$, independent of $N$, such that

$$
\left|\frac{1}{\varepsilon^{p}} \int_{\left(\varepsilon / 2^{N}, \varepsilon\right]} x^{p} v(\mathrm{~d} x)-\sum_{k=1}^{N}\left(\frac{1}{2^{p}}\right)^{k-1} \frac{c}{p}\left(1-\frac{1}{2^{p}}\right)\right|<\eta
$$

for every $0<\varepsilon<\delta$. Thus, letting $N \rightarrow \infty$, we arrive at condition 1 .

Remark 2.2. When $p \in \mathbb{N}$, conditions 1 and 2 of Proposition 2.1 can be rewritten as follows: $\lim _{\varepsilon \downarrow 0} \kappa_{p, \varepsilon}=\kappa_{p}$ for all, respectively some, $p \in \mathbb{N}$, where $\kappa_{p, \varepsilon}$ and $\kappa_{p}$ are the $p$ th-order cumulants of the RVs $X_{\varepsilon}(1) / \varepsilon$ and $X_{\star}^{c}(1)$, respectively (cf. [16, p. 35]).

Remark 2.3. We can easily show that condition 3 of Proposition 2.1 cannot be weakened to $' \lim _{\varepsilon \downarrow 0} v((\varepsilon x, \varepsilon])=c \log (1 / x)$ for some $0<x<1$ '.

Remark 2.4. By Proposition $2.1, \mu(\varepsilon) / \varepsilon \rightarrow c$ if and only if $v((\varepsilon x, \varepsilon]) \rightarrow c \log (1 / x)$ for all $0<x<1$, where $0<c<\infty$. It is useful to note that the same is true also for $c=0$. The 'if' part can be proved using the same technique as in step 2 of Proposition 2.1 (for $p=1$ ), and the 'only if' part follows from $\int_{(\varepsilon x, \varepsilon]} v(\mathrm{~d} u) \leq x^{-1} \varepsilon^{-1} \int_{(\varepsilon x, \varepsilon]} u v(\mathrm{~d} u)$. The last inequality also shows that $\mu(\varepsilon) / \varepsilon \rightarrow \infty$ if $\nu((\varepsilon x, \varepsilon]) \rightarrow \infty$ for some $0<x<1$. However, the inverse implication is not true. As an example, let $X$ be the subordinator of Example 3.11, below, for which it holds that $\mu(\varepsilon) / \varepsilon \rightarrow \infty$. For any $0<x<1$, we have, for sufficiently large and even $n, v\left(\left(\varepsilon_{n} x, \varepsilon_{n}\right]\right)=0$, where $\varepsilon_{n}=1 /\left(n 2^{n^{2}}\right)$, proving our claim.

The most important examples of subordinators $X \in \mathfrak{X}_{\star}$ arise from Corollary 2.3. Yet, there are also interesting examples where the Lévy measure is discrete.

Proposition 2.2. Let $\varphi$ be a strictly decreasing differentiable function on $\left[k_{0}, \infty\right), k_{0} \in \mathbb{N}$, such that $\varphi(x) \rightarrow 0$ and $\varphi(x+1) / \varphi(x) \rightarrow 1$ as $x \rightarrow \infty$. Furthermore, suppose that $\lambda$ is a monotone decreasing continuous function on $\left[k_{0}, \infty\right)$, satisfying

$$
c:=\lim _{x \rightarrow \infty} \frac{\varphi(x) \lambda(x)}{-\varphi^{\prime}(x)} \in(0, \infty)
$$

Let the Lévy measure of $X$ be concentrated on the set $\left\{\varphi(m): m=k_{0}, k_{0}+1, \ldots\right\}$, with $\nu(\{\varphi(m)\})=\lambda(m)$. Then $X_{\varepsilon} / \varepsilon \Rightarrow X_{\star}^{c}$ as $\varepsilon \downarrow 0$.

The following pairs of $\varphi$ and $\lambda$ satisfy the above conditions. In all cases, $c=\theta / r$.

- $\varphi(x)=1 / \log ^{r}(x), \lambda(x)=\theta /(x \log (x)), r>0$.

- $\varphi(x)=1 /\left(\log ^{q}(x) x^{r}\right), \lambda(x)=\theta / x, r>0, q \in \mathbb{R}$.

- $\varphi(x)=x^{q} \mathrm{e}^{-x^{r}}, \lambda(x)=\theta x^{r-1}, 0<r<1, q \in \mathbb{R}$.

Proof of Proposition 2.2. Clearly, $\varphi$ and $\lambda$ are both strictly positive on $\left[k_{0}, \infty\right)$. From (2.7), it follows easily that $\int_{k_{0}}^{\infty} \varphi(x) \lambda(x) \mathrm{d} x<\infty$ and $\int_{k_{0}}^{\infty} \lambda(x) \mathrm{d} x=\infty$. Since $\varphi \lambda$ and $\lambda$ are monotone, it also holds that $\sum_{m=k_{0}}^{\infty} \varphi(m) \lambda(m)<\infty$ and $\sum_{m=k_{0}}^{\infty} \lambda(m)=\infty$. Thus, $v$ is a valid Lévy measure for $X$. Now let $\varphi(m+1) \leq \varepsilon<\varphi(m), m \geq k_{0}$ integer. Hence, $\mu(\varepsilon)=\sum_{i=m+1}^{\infty} \varphi(i) \lambda(i)$, from which it follows that

$$
\frac{\varphi(m+1)}{\varphi(m)} \frac{\int_{m+1}^{\infty} \varphi(x) \lambda(x) \mathrm{d} x}{\varphi(m+1)}<\frac{\mu(\varepsilon)}{\varepsilon}<\frac{\varphi(m)}{\varphi(m+1)} \frac{\int_{m}^{\infty} \varphi(x) \lambda(x) \mathrm{d} x}{\varphi(m)} .
$$


Under assumptions (2.7) and $\varphi(m+1) / \varphi(m) \rightarrow 1$, we have, using l'Hôpital's rule, $\mu(\varepsilon) / \varepsilon \rightarrow c$ as $\varepsilon \downarrow 0$. Thus, $X_{\varepsilon} / \varepsilon \Rightarrow X_{\star}^{c}$.

Corollary 2.4. Let $\varphi, \lambda$, and $c$ be as in Proposition 2.2, and let $N_{n, i}$ be independent Poisson processes with rates $\lambda(n+i)$. Define a sequence $X_{n}=\left\{X_{n}(t): t \geq 0\right\}$ of processes by

$$
X_{n}(t)=\sum_{i=0}^{\infty} \frac{\varphi(n+i)}{\varphi(n)} N_{n, i}(t), \quad n=k_{0}, k_{0}+1, \ldots
$$

Then $X_{n} \Rightarrow X_{\star}^{c}$ as $n \rightarrow \infty$. In particular, $\sum_{i=0}^{\infty}(n /(n+i))^{r} N_{n, i} \Rightarrow X_{\star}^{\theta / r}$ for all $r>0$, where the $N_{n, i}$ have rates $\theta /(n+i)$.

Proof. Let $X$ be as in Proposition 2.2, and set $\varepsilon_{n}=\varphi(n)$. Thus, in particular, $X_{\varepsilon_{n}} / \varepsilon_{n} \Rightarrow X_{\star}^{c}$ as $n \rightarrow \infty$. The jump sizes of $X_{\varepsilon_{n}} / \varepsilon_{n}$ are $\varphi(n+i) / \varphi(n), i=0,1,2, \ldots$, and their rate is $\lambda(n+i)$. Hence, $X_{\varepsilon_{n}} / \varepsilon_{n}$ has the same law as $X_{n}$, and the claim follows.

Recall the discussion in Section 1 on the normal approximation of $X_{\varepsilon}$. We claimed the following.

Proposition 2.3. The weak convergence (1.7) implies that $\sigma(\varepsilon) / \mu(\varepsilon) \rightarrow 0$ and, in particular, that $X_{\varepsilon} / \mu(\varepsilon)$ converges weakly to the unit drift process.

Proof. Suppose by contradiction that (1.7) holds but $\sigma(\varepsilon) / \mu(\varepsilon) \nrightarrow \rightarrow 0$. Then there exists some $a>0$ and a sequence $\varepsilon_{n} \downarrow 0$ such that $\sigma\left(\varepsilon_{n}\right) / \mu\left(\varepsilon_{n}\right) \geq a$ for all $n$. It follows that $\left(X_{\varepsilon_{n}}(1)-\mu\left(\varepsilon_{n}\right)\right) / \sigma\left(\varepsilon_{n}\right) \geq-1 / a$, in contradiction to (1.7). The rest of the assertion is now evident by dividing the numerator and denominator of the left-hand side of (1.7) by $\mu(\varepsilon)$.

Since $X \in \mathfrak{X}_{\star}^{c}$ implies that $X_{\varepsilon} / \mu(\varepsilon) \Rightarrow c^{-1} X_{\star}^{c}$ as $\varepsilon \downarrow 0$, we obtain the following result.

Corollary 2.5. The normal approximation of small jumps is not valid for processes in $\mathfrak{X}_{\star}$.

Suppose now that $v$ is continuous near the origin, and recall the statement after (1.9). Since $\sigma^{2}(\varepsilon) / \varepsilon^{2} \leq \mu(\varepsilon) / \varepsilon, \mu(\varepsilon) / \varepsilon \rightarrow \infty$ is a necessary condition for (1.7) to hold. (Alternatively, this fact is a consequence of Proposition 2.3 combined with Theorem 2.1.) However, it is not a sufficient condition, as Example 3.11, below, shows.

The following proposition is important, among others, from a simulation point of view (as will be explained in Section 4). It also inspired Theorem 2.2, below.

Proposition 2.4. For any $X \in \mathfrak{X}_{\star}^{c}$, it holds that

$$
\nu((\varepsilon, \infty)) \sim c \log \left(\frac{1}{\varepsilon}\right) \quad \text { as } \varepsilon \downarrow 0 .
$$

Proof. It suffices to prove this for $v((\varepsilon, 1])$. Define $a_{k}=v\left(\left(2^{-k}, 2^{-k+1}\right]\right)-c \log (2)$ and partition $\left(2^{-n}, 1\right]$ as $\bigcup_{k=1}^{n}\left(2^{-k}, 2^{-k+1}\right]$ to obtain $\left|v\left(\left(2^{-n}, 1\right]\right)-n c \log (2)\right| \leq \sum_{k=1}^{n}\left|a_{k}\right|$. By condition 3 of Proposition 2.1, $\left|a_{n}\right| \rightarrow 0$ as $n \rightarrow \infty$, and, hence, $n^{-1} \sum_{k=1}^{n}\left|a_{k}\right| \rightarrow 0$ also. Thus, $v\left(\left(2^{-n}, 1\right]\right) \sim n c \log (2)$ as $n \rightarrow \infty$. Now write $2^{-(n+1)} \leq \varepsilon<2^{-n}$ to conclude that $v((\varepsilon, 1]) /(c \log (1 / \varepsilon))$ is bounded from above and below by $v\left(\left(2^{-(n+1)}, 1\right]\right) /(n c \log (2))$ and $v\left(\left(2^{-n}, 1\right]\right) /((n+1) c \log (2))$, respectively, and so the proposition follows.

As pointed out by the referee, the second example in Example 3.2, below, shows that (2.8) is not a sufficient condition for $X \in \mathfrak{X}_{\star}^{c}$. 
In the particular case where $X$ is a $\Gamma_{c, \lambda}$ process, $v((\varepsilon, \infty))$ can be found explicitly, using a standard representation of the function $E_{1}(s)=\int_{s}^{\infty} \mathrm{e}^{-u} u^{-1} \mathrm{~d} u$, to be

$$
v((\varepsilon, \infty))=c\left(\log \left(\frac{1}{\lambda \varepsilon}\right)-\gamma+\sum_{k=1}^{\infty} \frac{(-1)^{k+1}(\lambda \varepsilon)^{k}}{k ! k}\right),
$$

where $\gamma$ here and below stands for Euler's constant.

For completeness, we now state and prove the following simple yet powerful result.

Theorem 2.2. Suppose that $X \in \mathfrak{X}_{\star}^{c}$. Fix $t>0$, and define

$$
K(x ; t)=\exp \left[t\left(c \log \left(\frac{1}{x}\right)-v((x, 1])\right)\right], \quad x \in(0,1) .
$$

Then $K(\cdot ; t)$ is slowly varying at 0 , and it holds that

$$
\mathrm{P}(X(t) \leq x) \sim \frac{\exp [t(-\gamma c-v((1, \infty)))]}{\Gamma(c t+1)} x^{c t} K(x ; t) \quad \text { as } x \downarrow 0 .
$$

Proof. By condition 3 of Proposition 2.1, both $\mathrm{e}^{-t v((a x, x])}$ if $a<1$ and $\mathrm{e}^{t v((x, a x])}$ if $a \geq 1$ converge to $\mathrm{e}^{t c \log (a)}$ as $x \downarrow 0$. It follows that $K(a x ; t) / K(x ; t) \rightarrow 1$ as $x \downarrow 0$, for any fixed $a>0$. That is, $K(\cdot ; t)$ is slowly varying at 0 . In order to conform to our setting, we now replace $x$ by $\varepsilon$. Letting $\mathcal{A}$ denote the event that $X$ has no jump larger than $\varepsilon$ before time $t$, whose probability is equal to $\exp [-t v((\varepsilon, \infty))]$, we obtain

$$
\begin{aligned}
\mathrm{P}(X(t) \leq \varepsilon) & =\mathrm{P}(X(t) \leq \varepsilon, \mathcal{A}) \\
& =\mathrm{P}(\mathcal{A}) \mathrm{P}\left(X_{\varepsilon}(t) \leq \varepsilon\right) \\
& =\mathrm{P}\left(\frac{X_{\varepsilon}(t)}{\varepsilon} \leq 1\right) \exp [-t v((1, \infty))] \varepsilon^{c t} K(\varepsilon ; t) .
\end{aligned}
$$

Since $X_{\varepsilon}(t) / \varepsilon \stackrel{\mathrm{D}}{\rightarrow} X_{\star}^{c}(t)$, it remains to show that $\mathrm{P}\left(X_{\star}^{c}(t) \leq 1\right)=\exp [-\gamma c t] / \Gamma(c t+1)$. In fact, $\mathrm{P}\left(X_{\star}^{c}(t) \leq y\right)=\exp [-\gamma c t] y^{c t} / \Gamma(c t+1)$ for all $y \in[0,1]$; cf., e.g. (4.21), below.

Our presentation of Theorem 2.2 is deliberate. For if $X$ in this theorem is moreover selfdecomposable (see Example 3.1, below) with underlying function $k(k(0+)=c)$, then the definition of $K(x ; t)$ becomes

$$
K(x ; t)=\exp \left[t \int_{x}^{1} \frac{c-k(u)}{u} \mathrm{~d} u\right], \quad x \in(0,1),
$$

and $v((1, \infty))$ is equal to $\int_{1}^{\infty} k(x) x^{-1} \mathrm{~d} x$, and so, by setting $t=1$ in (2.10), we actually get the asymptotic formula, Equation (53.22), of [14] for the DF of such self-decomposable distributions, whose proof essentially constitutes the bulk of the proof of Theorem 53.6 of [14], which provides an analogous formula for the density function. It is worth mentioning that the constant $\int_{0}^{1}\left(\mathrm{e}^{-x}-1\right) x^{-1} \mathrm{~d} x+\int_{1}^{\infty} \mathrm{e}^{-x} x^{-1} \mathrm{~d} x$ appearing in the constants $\kappa$ of $[14$, Theorems 53.6 and 53.8] is merely the constant $-\gamma$ (appearing in our (2.10)). We also note that if $X$ is a $\Gamma_{c, \lambda}$ process then $\mathrm{P}(X(t) \leq x) \sim \lambda^{c t} x^{c t} / \Gamma(c t+1)$ as $x \downarrow 0$, which is readily seen from the corresponding density function. 


\section{Examples}

Example 3.1. Let $X$ be a nonzero pure-jump self-decomposable subordinator (see [14] for the definition and properties of self-decomposable distributions and processes). It is characterized by an absolutely continuous Lévy measure with density

$$
\rho(x)=\frac{k(x)}{x} \mathbf{1}_{(0, \infty)}(x),
$$

where $k(x)$ (nonnegative and satisfying $\left.\int_{0}^{\infty}(x \wedge 1)(k(x) / x) \mathrm{d} x<\infty\right)$ is monotone decreasing on $(0, \infty)$ with $k(0+) \in(0, \infty]$ (cf. [14, Corollary 15.11]). By Corollary 2.1, $X_{\varepsilon} / \mu(\varepsilon)$ converges weakly to the unit drift process if and only if $k(0+)=\infty$ and to $c^{-1} X_{\star}^{c}$ if and only if $k(0+)=c<\infty$; the latter holds if and only if $X_{\varepsilon} / \varepsilon \Rightarrow X_{\star}^{c}$. If $k(0+)=\infty$, the normal approximation of small jumps is valid $\left(\sigma^{2}(\varepsilon) / \varepsilon^{2} \rightarrow \infty\right)$. This is the case, for example, if $X$ is a (strictly) $\alpha$-stable subordinator, $0<\alpha<1$, i.e. it has Lévy density $\rho(x)=$ $b x^{-(1+\alpha)} \mathbf{1}_{(0, \infty)}(x), b>0$. The primary examples for the case $k(0+)=c<\infty$ are the process $a X_{\star}^{c}(a>0)$ and the $\Gamma_{c, \lambda}$ process. From the fact that $a^{\prime} X_{\star}^{c}$ has Lévy measure $\mathbf{1}_{\left(0, a^{\prime}\right]}(x) c x^{-1} \mathrm{~d} x$ $\left(a^{\prime}>0\right)$, it follows immediately that

$$
\left(a X_{\star}^{c}\right)_{\varepsilon} \stackrel{\text { LAW }}{=} \varepsilon X_{\star}^{c} \quad \text { for all } 0<\varepsilon \leq a .
$$

Thus, in some sense, the small jump part of $a X_{\star}^{c}$ is not approximated at all. The gamma case will be worked out in detail in the next section.

Example 3.2. We have already noted that $X_{\varepsilon} / \varepsilon \Rightarrow X_{\star}^{c}$ as $\varepsilon \downarrow 0$ can hold with a Lévy density $\rho$ not satisfying $\lim _{x \downarrow 0} x \rho(x)=c$. As a nontrivial example, let $\rho$ satisfy $\rho(x)=(1+$ $\sin (1 / x))(c / x)$ near $0+$, and apply condition 3 of Proposition 2.1. On the other hand, if we take $\rho(x)=(1+\sin (m \log (x)))(c / x)$ near $0+$, which somewhat resembles the previous one if $m$ is large, then $X_{\varepsilon} / \varepsilon$ fails to converge (to any law), and, moreover, the same holds even for $X_{\varepsilon} / \mu(\varepsilon)$. This assertion follows immediately from Corollary 2.2 and Theorem 2.1, using the fact that $\mu(\varepsilon) / \varepsilon$ in this case does not converge to a limit in $[0, \infty]$ (which can be verified by explicit integration or by virtue of Remark 2.4 , showing that $v((\varepsilon x, \varepsilon])$ does not have a limit for some $0<x<1$ ).

We now introduce an interesting class of subordinators for which $X_{\varepsilon} / \mu(\varepsilon)$ converges weakly to the zero process. Example 3.4, below, is some discrete counterpart.

Example 3.3. Suppose that $X$ has Lévy density $\rho$ satisfying $\rho(x)=x^{-1} L(x)$ on some finite interval $(0, A)$, where $L$ is slowly varying at 0 with $\lim _{x \downarrow 0} L(x)=0$ and, as required, $\int_{0}^{\delta} x^{-1} L(x) \mathrm{d} x=\infty$ for all $\delta \in(0, A)$. We will not take advantage of the slow variation of $L$ here. The typical examples, however, possess this property. Define $\log _{1}(x)=\log (x)$ and, recursively, $\log _{k}(x)=\log \left(\log _{k-1}(x)\right)$. Examples of functions $L$ that satisfy the above conditions are $L(x)=|\log (x)|^{-p}, 0<p \leq 1, L(x)=\left[\log _{k}(|\log (x)|)\right]^{-r}, k \in \mathbb{N}, r>0$, and $L(x)=|\log (x)|^{-p}\left[\log _{k}(|\log (x)|)\right]^{-r}, 0<p \leq 1, k \in \mathbb{N}, 0<r \leq 1$ or $r>0$ according as $p=1$ or $p<1$, respectively. Since $\lim _{x \downarrow 0} x \rho(x)=0, X_{\varepsilon} / \mu(\varepsilon)$ converges weakly to the zero process.

Example 3.4. Suppose that $X$ has Lévy measure concentrated on the set $\left\{\mathrm{e}^{-m}: m \in \mathbb{N}\right\}$ such that $v\left(\left\{\mathrm{e}^{-m}\right\}\right)=1 / h(m)$ for all sufficiently large $m$, where $h(u)=1 / L\left(\mathrm{e}^{-u}\right)$ with $L$ as in Example 3.3. Furthermore, assume that $h$ is increasing. (Note that $\int^{\infty}(1 / h(u)) \mathrm{d} u=\infty$ and, hence, $v$ is infinite.) Examples of such functions $h$ are $h(u)=u^{p}, 0<p \leq 1$, 
$h(u)=\left[\log _{k}(u)\right]^{r}, k \in \mathbb{N}, r>0$, and $h(u)=u^{p}\left[\log _{k}(u)\right]^{r}, 0<p \leq 1, k \in \mathbb{N}$, $0<r \leq 1$ or $r>0$ according as $p=1$ or $p<1$, respectively. It can be shown that $\int_{0}^{\varepsilon / \mathrm{e}} L(x) \mathrm{d} x<\mu(\varepsilon)<\int_{0}^{\mathrm{e} \varepsilon} L(x) \mathrm{d} x$. In particular, $\mu(\varepsilon) / \varepsilon \rightarrow 0$.

We now give a discrete counterpart of the $\alpha$-stable case mentioned in Example 3.1.

Example 3.5. Fix $0<\alpha<1$ and $b>0$, and let the Lévy measure of $X$ be given by $v(\{x\})=(b / \alpha) \mathbf{1}_{\left\{m^{-1 / \alpha}: m \in \mathbb{N}\right\}}(x)$. It can be shown that $\mu(\varepsilon) \sim(b /(1-\alpha)) \varepsilon^{1-\alpha}$ as $\varepsilon \downarrow 0$. The right-hand side is exactly $\mu(\varepsilon)$ of the $\alpha$-stable subordinator from Example 3.1, and the normal approximation of small jumps is valid (indeed, $\sigma^{2}(\varepsilon) / \varepsilon^{2} \rightarrow \infty$ ).

Examples 3.6-3.9, below, refer to the end of the proof of Theorem 2.1. Example 3.7 also refers to Remark 1.3.

Example 3.6. Let the Lévy measure of $X$ be given by $v(\{x\})=\mathbf{1}_{\left\{a^{-m}: m \in \mathbb{Z}_{+}\right\}}(x), a>1$. For any $a^{-(n+1)} \leq \varepsilon<a^{-n}, n \in \mathbb{Z}_{+}$, we find that $a^{-(n+m)} / \mu(\varepsilon)=(a-1) a^{-m}, m \in \mathbb{N}$, so that $\Pi_{[\mu(\varepsilon)]}$ is given by $\Pi_{[\mu(\varepsilon)]}(\{x\})=\mathbf{1}_{\left\{(a-1) a^{-m}: m \in \mathbb{N}\right\}}(x)$. Now let $\varepsilon \downarrow 0$. While $\mu(\varepsilon) / \varepsilon$ fluctuates in the interval $(1 /(a-1), a /(a-1)], X_{\varepsilon} / \mu(\varepsilon)$ trivially converges weakly since the underlying Lévy measure is constant across $\varepsilon$. With regard to Remark 1.1 and Corollary 2.2, we note that here $X_{\varepsilon_{n}} / \varepsilon_{n} \stackrel{\text { LAW }}{=} X$, where $\varepsilon_{n}=a^{-n}(n \in \mathbb{N})$, and so, in particular, $X_{\varepsilon_{n}} / \varepsilon_{n}$ converges weakly to a nonzero process other than $X_{\star}^{c}$.

Example 3.7. We now show the convergence of $X_{\varepsilon} / \mu(\varepsilon)$ in the case when $\mu(\varepsilon) / \varepsilon$ does not converge to a limit in $[0, \infty]$ and $\lim \sup _{\varepsilon \downarrow 0}(\mu(\varepsilon) / \varepsilon)=\infty$. From Theorem 2.1(d) we a priori know that the limit will be the unit drift process. Let the Lévy measure of $X$ be concentrated on the set $\left\{2^{-m^{2}}: m \in \mathbb{N}\right\}$ with $v\left(\left\{2^{-m^{2}}\right\}\right)=m$, and write $2^{-(n+1)^{2}} \leq \varepsilon<2^{-n^{2}}, n \in \mathbb{N}$. Set $\varepsilon_{n}=2^{-(n+1)^{2}}$ and obtain $\mu\left(\varepsilon_{n}\right) / \varepsilon_{n} \rightarrow \infty$ as $n \rightarrow \infty$; hence, as we have already seen in the proof of Theorem 2.1, $X_{\varepsilon_{n}} / \mu\left(\varepsilon_{n}\right)$ converges weakly to the unit drift process. The same holds for $X_{\varepsilon} / \mu(\varepsilon)$ as $\varepsilon \downarrow 0$ since $X_{\varepsilon} / \mu(\varepsilon)=X_{\varepsilon_{n}} / \mu\left(\varepsilon_{n}\right)$. Nevertheless, $\mu(\varepsilon) / \varepsilon$ fluctuates in $(0, \infty)$. As for Remark 1.3, we need to show that (1.8) holds. Fix $\kappa>0$ and consider the (nontrivial) case $\kappa \sigma(\varepsilon)<\varepsilon$. Then (1.8) follows by checking that $\varepsilon_{n}<\kappa \sigma(\varepsilon)(<\varepsilon)$ for sufficiently large $n$, where $\varepsilon$ and $\varepsilon_{n}$ are as above.

We now give the nonconvergent counterparts of Examples 3.6 and 3.7.

Example 3.8. Let the Lévy measure of $X$ be given by

$$
v(\{x\})=\mathbf{1}_{\left\{2^{-m}: m=0,2,4, \ldots\right\}}(x)+2 \cdot \mathbf{1}_{\left\{2^{-m}: m=1,3,5, \ldots\right\}}(x) .
$$

Set $\varepsilon_{n}=2^{-n}, n \in \mathbb{Z}_{+}$. If $n$ is even, we find that $2^{-(n+m)} / \mu\left(\varepsilon_{n}\right)=\frac{3}{8} 2^{-m}, m \in \mathbb{Z}_{+}$; hence, $\Pi_{\left[\mu\left(\varepsilon_{n}\right)\right]}$ is given by $\Pi_{\left[\mu\left(\varepsilon_{n}\right)\right]}(\{x\})=\mathbf{1}_{\left\{(3 / 8) 2^{-m}: m=0,2,4, \ldots\right\}}(x)+2 \cdot \mathbf{1}_{\left\{(3 / 8) 2^{-m}: m=1,3,5, \ldots\right\}}(x)$. If $n$ is odd, we find that $2^{-(n+m)} / \mu\left(\varepsilon_{n}\right)=\frac{3}{10} 2^{-m}, m \in \mathbb{Z}_{+}$, and, hence,

$$
\Pi_{\left[\mu\left(\varepsilon_{n}\right)\right]}(\{x\})=2 \cdot \mathbf{1}_{\left\{(3 / 10) 2^{-m}: m=0,2,4, \ldots\right\}}(x)+\mathbf{1}_{\left\{(3 / 10) 2^{-m}: m=1,3,5, \ldots\right\}}(x) .
$$

Thus, the sequences $X_{\varepsilon_{n}} / \mu\left(\varepsilon_{n}\right), n$ even, and $X_{\varepsilon_{n}} / \mu\left(\varepsilon_{n}\right), n$ odd, trivially converge weakly to different limits. In this example, $\mu(\varepsilon) / \varepsilon$ fluctuates in the interval $\left(\frac{4}{3}, \frac{10}{3}\right]$.

Example 3.9. Let the Lévy measure of $X$ be concentrated on the set $\left\{2^{-m}: m \in \mathbb{N}\right\}$, with $v\left(\left\{2^{-m}\right\}\right)=m$ if $\sqrt{m} \in \mathbb{N}$ and 1 otherwise. Set $\varepsilon_{n}=2^{-n}$. If $\sqrt{n} \in \mathbb{N}$ then $\mu\left(\varepsilon_{n}\right) / \varepsilon_{n}>$ $n(\rightarrow \infty)$, and so $X_{\varepsilon_{n}} / \mu\left(\varepsilon_{n}\right)$ converges weakly to the unit drift process. If $\sqrt{n-1} \in \mathbb{N}$, $X_{\varepsilon_{n}} / \mu\left(\varepsilon_{n}\right)$ converges weakly to a pure-jump subordinator, say $Y$, with Lévy measure $Q$ given 
by $Q(\{x\})=\mathbf{1}_{\left\{2^{-m}: m \in \mathbb{N}\right\}}(x)$. This is shown as follows. First observe that $\varepsilon_{n} / \mu\left(\varepsilon_{n}\right)<\frac{1}{2}$ (for all $n \in \mathbb{N})$. Hence, $\int_{(0, h]} x \Pi_{\left[\mu\left(\varepsilon_{n}\right)\right]}(\mathrm{d} x)=\mathrm{E}\left[X_{\varepsilon_{n}}(1) / \mu\left(\varepsilon_{n}\right)\right]=1$ for all $h>\frac{1}{2}$. Since also $\int_{(0, h]} x Q(\mathrm{~d} x)=1$, condition (i) from Section 1 is satisfied. Now assume that $\sqrt{n-1} \in \mathbb{N}$ and fix $M \in \mathbb{N}$. Let $x_{m}=2^{-(n+m)} / \mu\left(\varepsilon_{n}\right), m=0,1, \ldots, M$, be the $M+1$ largest mass points of $\Pi_{\left[\mu\left(\varepsilon_{n}\right)\right]}$. Then $\Pi_{\left[\mu\left(\varepsilon_{n}\right)\right]}\left(\left\{x_{m}\right\}\right)=1$ if $n$ is sufficiently large and $x_{m}=2^{-(m+1)} r_{n}$, where $r_{n}$ can be made arbitrarily close to 1 by increasing $n$. We conclude that condition (ii) from Section 1 holds as well, that is, $\Pi_{\left[\mu\left(\varepsilon_{n}\right)\right]} \stackrel{\mathrm{v}}{\rightarrow} Q$. Thus, $X_{\varepsilon_{n}} / \mu\left(\varepsilon_{n}\right) \Rightarrow Y(\sqrt{n-1} \in \mathbb{N})$. In this example, $\mu(\varepsilon) / \varepsilon$ fluctuates in $(1, \infty)$.

The following example further illustrates the point made in Remark 1.1 about the significance of $\varepsilon$ being a continuous parameter.

Example 3.10. Define $B=\bigcup_{m \in\{1,3,5, \ldots\}}\left(\alpha_{m+1}, \alpha_{m}\right], \alpha_{m}=1 / 2^{m^{2}}$, and let $X$ have Lévy measure $v(\mathrm{~d} x)=\mathbf{1}_{B}(x)(c / x) \mathrm{d} x$. Let $\varepsilon_{n}=\alpha_{n} / n, n$ odd. For any $0<a<b<\infty$, $\nu\left(\left(\varepsilon_{n} a, \varepsilon_{n} b\right]\right)=c \log (b / a)$ for all sufficiently large $n$; moreover, $\varepsilon_{n}^{-p} \int_{\left(0, \varepsilon_{n}\right]} x^{p} v(\mathrm{~d} x) \rightarrow c / p$ (as $n \rightarrow \infty$, odd) for every $p>0$. It follows, in particular, that $X_{\varepsilon_{n}} / \varepsilon_{n} \stackrel{\left.\varepsilon_{n}\right]}{\Rightarrow} X_{\star}^{c}$. However, for any even $n$, we have $v\left(\left(\alpha_{n+1}, \alpha_{n}\right]\right)=0$, and, hence, obviously, $X_{\varepsilon} / \varepsilon \nRightarrow X_{\star}^{c}$. With $\varepsilon_{n}$ as above, we also have $X_{\varepsilon_{n}} / \mu\left(\varepsilon_{n}\right) \Rightarrow c^{-1} X_{\star}^{c}$, but $X_{\varepsilon} / \mu(\varepsilon) \nRightarrow c^{-1} X_{\star}^{c}$.

Assume that $v$ is continuous near the origin. The following example serves as a counter example showing that $\mu(\varepsilon) / \varepsilon \rightarrow \infty$-and, hence, also the convergence of $X_{\varepsilon} / \mu(\varepsilon)$ to the unit drift process - is not a sufficient condition for (1.7) to hold, though necessary.

Example 3.11. Define $\alpha_{m}=1 / 2^{m^{2}}, m \in \mathbb{N}$, and let the Lévy measure of $X$ be concentrated on $\bigcup_{m \in\{1,3,5, \ldots\}}\left(\alpha_{m+1}, \alpha_{m}\right]$ with $v(\mathrm{~d} x)=2^{m^{2}+\beta m} \mathrm{~d} x$ on $\left(\alpha_{m+1}, \alpha_{m}\right], \beta \in(2,4)$ fixed. (Here $v$ is indeed an infinite Lévy measure of a subordinator.) We claim that $\mu(\varepsilon) / \varepsilon \rightarrow \infty$ as $\varepsilon \downarrow 0$. A sketch of the proof is as follows. Let $\alpha_{n+1} \leq \varepsilon<\alpha_{n}$. If $n$ is even, show that $\int_{\alpha_{n+2}}^{\alpha_{n+1}} x v(\mathrm{~d} x) / \varepsilon \rightarrow \infty$ as $n \rightarrow \infty$. If $n$ is odd, write $\varepsilon$ as $\varepsilon=1 / 2^{(n+\theta)^{2}}$ with $\theta \in(0,1]$. Then consider the expressions $\int_{\alpha_{n+1}}^{\varepsilon} x v(\mathrm{~d} x) / \varepsilon$ if $\theta \leq(6-\beta) / 4$ and $\int_{\alpha_{n+3}}^{\alpha_{n+2}} x v(\mathrm{~d} x) / \varepsilon$ if $\theta>(6-\beta) / 4$ to conclude that $\mu(\varepsilon) / \varepsilon \rightarrow \infty$. To show that (1.7) fails, it suffices to show that $\sigma^{2}(\varepsilon) / \varepsilon^{2} \nrightarrow \infty$. Indeed, if $\varepsilon_{n}=\alpha_{n}$ with $n$ even, then $\sigma^{2}\left(\varepsilon_{n}\right) / \varepsilon_{n}^{2} \rightarrow 0$.

Example 3.12. A variance gamma process $Z$ can be represented as $Z=X_{1}-X_{2}$, where $X_{1}$ and $X_{2}$ are independent $\Gamma_{c, \lambda_{1}}$ and $\Gamma_{c, \lambda_{2}}$ processes, respectively (cf. [12, Equation (14)]). We conclude that $Z$ admits the following approximation:

$$
Z(t) \cong \varepsilon\left[X_{\star 1}^{c}(t)-X_{\star 2}^{c}(t)\right]+X_{1}^{\varepsilon}(t)-X_{2}^{\varepsilon}(t) \text { for } \varepsilon \text { small, }
$$

where the $X_{\star 1,2}^{c}$ have the same law as $X_{\star}^{c}$, the $X_{1,2}^{\varepsilon}$ are compound Poisson processes with respective Lévy densities $\mathbf{1}_{(\varepsilon, \infty)}(x) c \mathrm{e}^{-\lambda_{1,2} x} x^{-1}$, and the four processes are independent.

Recall the convergence result (1.11). Its analogue for the variance gamma process is given by [5, Example 5.2(b)], adapted to our setting, as follows:

$$
\frac{Z_{\varepsilon}-\tilde{A}_{\varepsilon}}{\tilde{\sigma}(\varepsilon)} \Rightarrow \tilde{Y}^{c} \quad \text { as } \varepsilon \downarrow 0,
$$

where $Z_{\varepsilon}$ consists of those jumps of $Z$ not exceeding $\varepsilon$ in absolute value, $\tilde{A}_{\varepsilon}$ is the drift process defined by $\tilde{A}_{\varepsilon}(t)=\mathrm{e}\left[Z_{\varepsilon}(t)\right], \tilde{\sigma}^{2}(\varepsilon)=\operatorname{var}\left[Z_{\varepsilon}(1)\right]$, and $\tilde{Y}^{c}$ is a pure-jump symmetric Lévy process with lévy measure $\tilde{\pi}(\mathrm{d} x)=\mathbf{1}_{\left\{x: 0<x^{2} \leq c^{-1}\right\}}(x) c|x|^{-1} \mathrm{~d} x$. the connection with our result, $Z_{\varepsilon} / \varepsilon \rightarrow X_{\star 1}^{c}-X_{\star 2}^{c}$, is made similarly as in the gamma case. 


\section{Analysis of approximations for a special subclass of $\mathfrak{X}$}

We have already described briefly in Section 1 the main content of the present section. We begin with some preliminaries.

We denote by $\mathfrak{Y}^{c, \alpha}, c, \alpha>0$, the class of pure-jump subordinators with Lévy density $\rho$ such that $\delta(x):=c-x \rho(x)$ equals $x^{\alpha} L(x)$ for some function $L$ that is slowly varying at 0 (to put it otherwise, $\delta(x)$ is regularly varying at 0 with index $\alpha$ ). We recall that $L$ is said to be slowly varying at 0 if it is a positive measurable function, defined on some finite interval $(0, A)$, such that $L(b x) / L(x) \rightarrow 1$ as $x \downarrow 0$ for all $b>0$. Accordingly, $\delta(x)$ is defined for $x \in(0, A)$. An elementary property of such functions $L$ is that $x^{\alpha} L(x) \rightarrow 0$ and $x^{-\alpha} L(x) \rightarrow \infty(x \downarrow 0)$. Thus, in particular, $\mathfrak{Y}^{c, \alpha} \subset \mathfrak{X}_{\star}^{c}$ (by Corollary 2.3). The class $\mathfrak{Y}^{c, \alpha}$ is significant primarily since it contains any pure-jump self-decomposable subordinator whose underlying function $k$ satisfies $k(x)=c-x^{\alpha} L(x)(x \in(0, A))$ with $L$ slowly varying at 0 , in particular the $\Gamma_{c, \lambda}$ process where $k(x)=c \mathrm{e}^{-\lambda x}$ (and, hence, $\delta(x)=x^{1} L(x)$, with $L(x)=c \lambda+O(x)$ as $x \downarrow 0$ ).

Given $X \in \mathfrak{Y}^{c, \alpha}$ with Lévy density $\rho$, we let $\tilde{X}(\cdot ; \varepsilon)$ be its approximation defined by

$$
\tilde{X}(t ; \varepsilon)=\varepsilon X_{\star}^{c}(t)+X^{\varepsilon}(t), \quad t \geq 0,
$$

where $X^{\varepsilon}:=X-X_{\varepsilon}$ (compound Poisson with Lévy density $\rho \mathbf{1}_{(\varepsilon, \infty)}$ ) is independent of $X_{\star}^{c}$. We always assume that $\varepsilon<A$. The DFs of $X(t)$ and $\tilde{X}(t ; \varepsilon)$ are denoted by $F(\cdot ; t)$ and $\tilde{F}(\cdot ; t ; \varepsilon)$, respectively. Since $X$ has an infinite absolutely continuous Lévy measure, $X(t)$ is absolutely continuous [14, Theorem 27.7]; so we let $f(\cdot ; t)$ denote its density. A central role in our analysis is played by a compound Poisson process, which we denote by $Y_{\varepsilon}$, such that $Y_{\varepsilon}$, $X_{\varepsilon}$, and $X^{\varepsilon}$ are mutually independent, and

$$
X_{\star}^{c}(t) \stackrel{\mathrm{D}}{=} \frac{X_{\varepsilon}(t)}{\varepsilon}+Y_{\varepsilon}(t) .
$$

Since $X_{\varepsilon} / \varepsilon$ has Lévy density $\varepsilon \rho(\varepsilon x)$ on $(0,1]$ (e.g. by (1.3)), it follows that $Y_{\varepsilon}$ has Lévy density $q_{\varepsilon}(x)=\mathbf{1}_{(0,1]}(x) \delta(\varepsilon x) x^{-1}$. Put another way, $Y_{\varepsilon}$ is compound Poisson with rate

$$
\Lambda(\varepsilon)=\int_{0}^{1} \frac{\delta(\varepsilon s)}{s} \mathrm{~d} s=\int_{0}^{\varepsilon} \frac{\delta(s)}{s} \mathrm{~d} s=\int_{0}^{\varepsilon} s^{\alpha-1} L(s) \mathrm{d} s \in(0, \infty)
$$

and jump distribution $J_{\varepsilon}(\mathrm{d} x)=q_{\varepsilon}(x) \Lambda(\varepsilon)^{-1} \mathrm{~d} x$ converging to beta $(\alpha, 1)$ as $\varepsilon \downarrow 0$; indeed, using (4.4), below, we show that $J_{\varepsilon}((0, x]) \rightarrow x^{\alpha}$ for all $0<x \leq 1$ fixed. By a suitable adaptation of [3, Proposition 1.5.10] (a part of Karamata's theorem), we have

$$
\int_{0}^{\varepsilon} s^{p} L(s) \mathrm{d} s \sim \frac{\varepsilon^{p+1}}{p+1} L(\varepsilon) \quad \text { as } \varepsilon \downarrow 0 \quad \text { for all } p>-1 .
$$

Letting $\Delta(x ; t ; \varepsilon)$ denote the difference $F(x ; t)-\tilde{F}(x ; t ; \varepsilon)$, we can now prove the following result.

Theorem 4.1. Suppose that $X \in \mathfrak{Y}^{c, \alpha}$, with $\delta(x)=x^{\alpha} L(x)$ on $(0, A)$. Fix any $x>0, t>0$, and $n \in \mathbb{N}$, and let $0<\varepsilon<A$. If the density $f(\cdot ; t)$ of $X(t)$ is bounded on $[x-n \varepsilon, x]$, then $\Delta(x ; t ; \varepsilon)$ (strictly positive) is bounded from above and below, respectively, by

$$
\begin{aligned}
& \sup _{u \in[x-\varepsilon, x]} f(u ; t) t \int_{0}^{\varepsilon} s^{\alpha} L(s) \mathrm{d} s+\sum_{k=2}^{n} \sup _{u \in[x-k \varepsilon, x]} f(u ; t) t^{k} \frac{2^{k-1}}{(k-1) !} \Lambda(\varepsilon)^{k} \varepsilon \\
& +F(x ; t) t^{n+1} \frac{2^{n}}{(n+1) !} \Lambda(\varepsilon)^{n+1}
\end{aligned}
$$


and

$$
\begin{aligned}
& \inf _{u \in[x-\varepsilon, x]} f(u ; t) t \int_{0}^{\varepsilon} s^{\alpha} L(s) \mathrm{d} s-\sum_{k=2}^{n} \sup _{u \in[x-k \varepsilon, x]} f(u ; t) t^{k} \frac{2^{k-1}}{(k-1) !} \Lambda(\varepsilon)^{k} \varepsilon \\
& -F(x ; t) t^{n+1} \frac{2^{n}}{(n+1) !} \Lambda(\varepsilon)^{n+1}-\sum_{k=n+2}^{\infty} \mathrm{e}^{-\Lambda(\varepsilon) t} \frac{[\Lambda(\varepsilon) t]^{k}}{k !} .
\end{aligned}
$$

If $f(\cdot ; t)$ is left continuous at $x$ then, in particular,

$$
\Delta(x ; t ; \varepsilon)=f(x ; t) t \frac{\varepsilon^{\alpha+1} L(\varepsilon)}{\alpha+1}+o\left(\varepsilon^{\alpha+1} L(\varepsilon)\right) \quad \text { as } \varepsilon \downarrow 0 .
$$

Proof. Let $x, t, n$, and $\varepsilon$ be as in the theorem. By $\varepsilon X_{\star}^{c}(t) \stackrel{\mathrm{D}}{=} X_{\varepsilon}(t)+\varepsilon Y_{\varepsilon}(t)$, we obtain

$$
\begin{aligned}
\tilde{F}(x ; t ; \varepsilon) & =\mathrm{P}\left(X_{\varepsilon}(t)+\varepsilon Y_{\varepsilon}(t)+X^{\varepsilon}(t) \leq x\right) \\
& =\mathrm{P}\left(X(t)+\varepsilon Y_{\varepsilon}(t) \leq x\right) \\
& =\sum_{k=0}^{\infty} \mathrm{P}\left(X(t)+\varepsilon\left[\xi_{1}+\cdots+\xi_{k}\right] \leq x\right) P_{k}(\Lambda(\varepsilon) t),
\end{aligned}
$$

where the $\xi_{i}$ are i.i.d. with distribution $J_{\varepsilon}$ (independent also of $\left.X(t)\right)$ and the $P_{k}(\Lambda(\varepsilon) t)$ are the Poisson probabilities with mean $\Lambda(\varepsilon) t$. We prove only the upper bound, (4.5). The proof of the lower bound, (4.6), is almost analogous. We omit some details that can be easily worked out by the reader. Expanding the probabilities $P_{k}(\Lambda(\varepsilon) t)$ in powers of $\Lambda(\varepsilon) t$ and using the inequality $\mathrm{e}^{-u}>\sum_{k=0}^{m}(-1)^{k} u^{k} / k !, m$ odd, $u>0$, we find that

$$
\begin{aligned}
\Delta(x ; t ; \varepsilon)< & \Lambda(\varepsilon) t\left[\mathrm{P}(X(t) \leq x)-\mathrm{P}\left(X(t)+\varepsilon \xi_{1} \leq x\right)\right] \\
& +\sum_{k=2}^{n}[\Lambda(\varepsilon) t]^{k} \sum_{j=0}^{k}(-1)^{k-1+j} c_{k, j} \mathrm{P}\left(X(t)+\varepsilon\left[\xi_{1}+\cdots+\xi_{j}\right] \leq x\right) \\
& +[\Lambda(\varepsilon) t]^{n+1} \sum_{j=0}^{\lfloor n / 2\rfloor} c_{n+1,2 j+(n \bmod 2)} \mathrm{P}_{n, j}(x ; t ; \varepsilon),
\end{aligned}
$$

where $c_{k, j}=1 /(j !(k-j) !), k \geq 2, j=0, \ldots, k$, and the $\mathrm{P}_{n, j}(x ; t ; \varepsilon)$ are certain probabilities bounded from above by $F(x ; t)$. By conditioning on $\xi_{1}$, the first expression on the right-hand side of (4.8) equals $t \int_{0}^{1}[F(x ; t)-F(x-\varepsilon s ; t)](\delta(\varepsilon s) / s) \mathrm{d} s$, implying that it is bounded from above by $\sup _{u \in[x-\varepsilon, x]} f(u ; t) t \int_{0}^{\varepsilon} s^{\alpha} L(s) \mathrm{d} s$. To bound the second expression, we replace $\mathrm{P}\left(X(t)+\varepsilon\left[\xi_{1}+\cdots+\xi_{j}\right] \leq x\right)$ by $\mathrm{P}(X(t) \leq x)$ if $k-1+j$ is even and by $\mathrm{P}(X(t)+\varepsilon k \leq$ $x)$ if $k-1+j$ is odd; the fact that $\sum_{j \in\{0,1, \ldots, k\} \text { even }} c_{k, j}=\sum_{j \in\{0,1, \ldots, k\} \text { odd }} c_{k, j}=2^{k-1} / k$ ! then straightforwardly leads to the middle expression in (4.5) as an upper bound. Writing $\sum_{j=0}^{\lfloor n / 2\rfloor} c_{n+1,2 j+(n \bmod 2)}$ as $\sum_{j \in\{0,1, \ldots, n+1\}, n+j \text { even }} c_{n+1, j}\left(=2^{n} /(n+1) !\right)$ now leads to (4.5) as an upper bound for $\Delta(x ; t ; \varepsilon)$. Together with the lower bound, (4.6), and by virtue of (4.3) and (4.4) (note that $n$ is arbitrary), (4.7) follows immediately.

Remark 4.1. The bounds in (4.5) and (4.6) (and, hence, also (4.7)) may be subject to further refinement. In particular, if $f^{\prime}(\cdot ; t)$ exists and is bounded on $[x-2 \varepsilon, x]$, then we can show, with a little more work, that the expression

$$
[\Lambda(\varepsilon) t]^{2} \sum_{j=0}^{2}(-1)^{1+j} c_{2, j} \mathrm{P}\left(X(t)+\varepsilon\left[\xi_{1}+\cdots+\xi_{j}\right] \leq x\right)
$$


(cf. (4.8)) is bounded in absolute value by $\sup _{u \in[x-2 \varepsilon, x]}\left|f^{\prime}(u ; t)\right| t^{2} \varepsilon \Lambda(\varepsilon) \int_{0}^{\varepsilon} s^{\alpha} L(s) \mathrm{d} s$, which in turn is asymptotically equal to $\left|f^{\prime}(x ; t)\right| t^{2}\left[\varepsilon^{\alpha+1} L(\varepsilon)\right]^{2} /(\alpha(\alpha+1))$ as $\varepsilon \downarrow 0$ if $f^{\prime}(\cdot ; t)$ is left continuous at $x$. The smoothness of $f(\cdot ; t)$ will be discussed shortly.

Before we give a corollary of the above theorem concerning the special gamma case, we note a couple of general inequalities, starting with the following simple one:

$$
\Delta(x ; t ; \varepsilon)<\left(1-\mathrm{e}^{-t \Lambda(\varepsilon)}\right) F(x ; t), \quad \varepsilon \in(0, A),
$$

which holds for any $X \in \mathfrak{Y}^{c, \alpha}$ and $x, t>0$ (this follows from $\tilde{F}(x ; t ; \varepsilon)>\mathrm{P}\left(X(t)+\varepsilon Y_{\varepsilon}(t) \leq\right.$ $\left.x, Y_{\varepsilon}(t)=0\right)$ ). Note that the coefficient of $F(x ; t)$ is asymptotically $t \alpha^{-1} \varepsilon^{\alpha} L(\varepsilon)$ as $\varepsilon \downarrow 0$ and that uniformity in $x$ is established by dropping $F(x ; t)$. From (4.9), it follows that

$$
\Delta(x ; t ; \varepsilon)<\min \{t \Lambda(\varepsilon), F(x ; t)\} \leq t^{*} \Lambda(\varepsilon),
$$

where $t^{*}=t^{*}(x ; \varepsilon)$ is the (unique) solution $t$ of $t \Lambda(\varepsilon)=F(x ; t)$. By comparing $X(t)$ with the sum of $[t]$ independent copies of $X(1)$ and considering the central limit theorem, we would expect, in general and roughly speaking, appropriate values of $t^{*}$ as long as $x$ is not too large. Thus, uniformity in $t$ (for $x$ fixed) is established.

Corollary 4.1. Let $X$ be $a \Gamma_{c, \lambda}$ process. Fix any $x>0$ and $t>0$. Then,

$$
\Delta(x ; t ; \varepsilon)=\frac{\lambda^{c t+1} x^{c t-1} \mathrm{e}^{-\lambda x} c t}{2 \Gamma(c t)} \varepsilon^{2}+O\left(\varepsilon^{3}\right) \quad \text { as } \varepsilon \downarrow 0 .
$$

Proof. Here $\alpha=1, L(s)=c \lambda+O(s)$ as $s \downarrow 0$, and $f(y ; t)=\lambda^{c t} y^{c t-1} \mathrm{e}^{-\lambda y} / \Gamma(c t), y>0$. In particular, $\int_{0}^{\varepsilon} s^{\alpha} L(s) \mathrm{d} s=c \lambda \varepsilon^{2} / 2+O\left(\varepsilon^{3}\right)$ and $\Lambda(\varepsilon) \sim c \lambda \varepsilon$. Now, by (4.5) and (4.6), it suffices to note that $\sup _{u \in[x-\varepsilon, x]}|f(x ; t)-f(u ; t)| \leq \sup _{u \in[x-\varepsilon, x]}\left|f^{\prime}(u ; t)\right| \varepsilon$.

It is worth noting that in the gamma case above, $\Delta(x ; t ; \varepsilon)<F(x ; t) \lambda c t \varepsilon$ for all $x, t, \varepsilon>0$. This follows straightforwardly from (4.9) using the fact that $L(s)=c\left(1-\mathrm{e}^{-\lambda s}\right) s^{-1}, s>0$.

The regularity assumptions on the density $f(\cdot ; t)$ of $X(t)$ in Theorem 4.1 are mild and reasonable. Let $\mathfrak{S} \mathfrak{D}^{c}$ denote the class of pure-jump self-decomposable subordinators with $k(0+)=c \in(0, \infty)\left(k(x)\right.$ as in Example 3.1). Having in mind that the class $\mathfrak{Y}^{c, \alpha}$ is interesting primarily since it contains an important subclass of $\mathfrak{S D}^{c}$ (as already noted), the following result from [14] shows that much stronger regularity conditions on $f(\cdot ; t)$ are not too restrictive. Let $X \in \mathfrak{S D}^{c}$. From [14, Remark 28.7] we infer the following statement. If $0<c t \leq 1$ then $f(\cdot ; t)$ is continuous on $(0, \infty)$ but discontinuous at 0 . If $c t>1$ then, letting $n=\lceil c t\rceil-1$, $f(\cdot ; t)$ is of class $C^{n-1}$ on $\mathbb{R}$ and of class $C^{n}$ on $(0, \infty)$, but not of class $C^{n}$ on $\mathbb{R}$. Here, $C^{n}$ stands for the class of $n$ times continuously differentiable functions ( $C^{0}$ being the class of continuous functions).

For processes in $\mathfrak{S} \mathfrak{D}^{c} \cap \mathfrak{Y}^{c, \alpha}$ we have the following uniform result.

Corollary 4.2. Suppose that $X \in \mathfrak{S D}^{c} \cap \mathfrak{Y}^{c, \alpha}$. If $t=1 / c$ then $0<f(0+; t)<\infty$ and

$$
\sup _{x} \Delta(x ; t ; \varepsilon) \sim f(0+; t) t \frac{\varepsilon^{\alpha+1} L(\varepsilon)}{\alpha+1} \text { as } \varepsilon \downarrow 0 .
$$

If $t>1 / c$ is fixed then $f(\cdot ; t)$ has a global maximum at some point $a>0$ and

$$
\sup _{x} \Delta(x ; t ; \varepsilon) \sim f(a ; t) t \frac{\varepsilon^{\alpha+1} L(\varepsilon)}{\alpha+1} \text { as } \varepsilon \downarrow 0 .
$$


Proof. We know that $f(\cdot ; t)$ is continuous on $(0, \infty)$ and, moreover, on $\mathbb{R}$ if $t>1 / c$. By $[14$, Theorem 53.1], the distribution of $X(t)$ is unimodal, $X$ being in particular a self-decomposable process. The unimodality of $X(t)$ implies that $f(\cdot ; t)$ is monotone increasing on $(-\infty, a)$ and monotone decreasing on $(a, \infty)$, for some $a \geq 0$ (cf. [14, Definition 23.2]). Assume first that $c t=1$. Then the above holds with mode $a=0$ (cf. [14, (53.16)]). Moreover, it follows from Remark 53.10(ii) of [14] that $f(0+; t)$ in this case is finite or infinite according to whether $\int_{0}^{1}(1-t k(x)) x^{-1} \mathrm{~d} x$ is finite or infinite, respectively. (We note that, by Remark 53.10(i) of [14], $f(0+; t)=\infty$ if $c t<1$.) Since in our case $1-t k(x)=t x^{\alpha} L(x)$, say on $(0,1), f(0+; t)$ is finite. Equation (4.11) then follows from (4.5) and (4.6). Assume now that $c t>1$. Then, by unimodality, $f(\cdot ; t)$ has a global maximum at some point $a>0$, and so (4.12) follows from (4.5) and (4.6).

Corollary 4.3. Suppose that $X$ is a $\Gamma_{c, \lambda}$ process. If $t \geq 1 / c$ is fixed then

$$
\sup _{x} \Delta(x ; t ; \varepsilon) \sim \frac{\lambda^{2}(c t-1)^{c t-1} \mathrm{e}^{-(c t-1)} c t}{2 \Gamma(c t)} \varepsilon^{2} \text { as } \varepsilon \downarrow 0,
$$

where $0^{0}$ is understood as 1 when $c t=1$. Moreover, the coefficient of $\varepsilon^{2}$ on the right-hand side of (4.13) is asymptotically equal to $\lambda^{2} \sqrt{c t /(8 \pi)}$ as $t \rightarrow \infty$.

Proof. The case in which $t=1 / c$ follows immediately from (4.11). If $c t>1$, the density of $X(t)$ has a maximum at (the mode) $a=(c t-1) / \lambda$, and so (4.13) follows from (4.12). The rest of the assertion follows from $\Gamma(z) \sim \sqrt{2 \pi} z^{z-1 / 2} \mathrm{e}^{-z}, z \rightarrow \infty$.

Some discussion of simulation aspects is in order. The process $\tilde{X}(\cdot ; \varepsilon)$, which is basically easy to simulate, is an excellent choice for an approximating process to $X$, actually, regarding processes $X \in \mathfrak{X}$ in general. The above results already indicate this with respect to the class $\mathfrak{Y}^{c, \alpha}$ and, particularly, its subclass $\mathfrak{S D}^{c} \cap \mathfrak{Y}^{c, \alpha}$. For a general $X \in \mathfrak{X}_{\star}^{c}$, the associated compound Poisson process, $X^{\varepsilon}$, has rate $v((\varepsilon, \infty))$, which is asymptotically $c \log (1 / \varepsilon)$ as $\varepsilon \downarrow 0$ (Proposition 2.4), and, hence, relatively small in general. This is very advantageous from a computational point of view. (For comparison, for the $\alpha$-stable subordinator in Example 3.1, it holds that $v((\varepsilon, \infty))=b \alpha^{-1} \varepsilon^{-\alpha}$.) The small jump part, $X_{\varepsilon}$, is quite negligible by definition, as $X_{\varepsilon} \cong \varepsilon X_{\star}^{c}$. Yet, incorporating its approximation-which becomes significant as $t$ gets large enough-allows the choice of a relatively large $\varepsilon$. The approximation $X_{\varepsilon} \cong \varepsilon X_{\star}^{c}$ will be analyzed shortly.

At this point, it should be mentioned that there are a number of other methods by which a gamma process can be easily/efficiently simulated; see [4, Section 4.1] for some details. Yet, in light of our results, simulation of a $\Gamma_{c, \lambda}$ process according to (4.1) is advisable (note that $X^{\varepsilon}$ in this case has rate $v((\varepsilon, \infty))$ given by $(2.9))$.

The high quality of our results, throughout this section, owes heavily to their limited scope of application. At this point it is of interest to make a comparison with Theorem 3.1 of [2]. Let $X$ be a pure-jump subordinator with infinite Lévy measure $v$, and define its approximation $\hat{X}(\cdot ; \varepsilon)$ by the right-hand side of (1.10). Furthermore, set $\varphi(\varepsilon)=\sigma^{-3}(\varepsilon) \int_{(0, \varepsilon]} x^{3} v(\mathrm{~d} x)$. Then, by [2, Theorem 3.1],

$$
\sup _{x \in \mathbb{R}}|\mathrm{P}(\hat{X}(1 ; \varepsilon) \leq x)-\mathrm{P}(X(1) \leq x)| \leq 0.7975 \varphi(\varepsilon) .
$$

(The result, with appropriate modifications, is given there for any Lévy process.) Of course, (4.14) is designated for processes for which (1.7) is satisfied. If $X \in \mathfrak{X}_{\star}^{c}$, we obtain, from 
condition 1 of our Proposition $2.1, \varphi(\varepsilon) \rightarrow \sqrt{8 /(9 c)}$ as $\varepsilon \downarrow 0$, so (4.14) is useless in this case. By implicitly considering a process with Lévy measure $t v,(4.14)$ leads us to

$$
\sup _{x \in \mathbb{R}}|\mathrm{P}(\hat{X}(t ; \varepsilon) \leq x)-\mathrm{P}(X(t) \leq x)| \leq 0.7975 t^{-1 / 2} \varphi(\varepsilon) .
$$

This inequality has three useful properties. First, its scope of usefulness is very wide. In particular, it is appropriate when condition (1.9) is satisfied, since then $\varphi(\varepsilon) \rightarrow 0$ as $\varepsilon \downarrow 0$. Second, the result is uniform in $x$. Finally, note the decrease of the bound to 0 as $t \rightarrow \infty$. On the other hand, generally speaking, for $t$ fixed, the bound is asymptotically not as good as one would intuitively expect. Indeed, consider the typical case where $X$ has Lévy density $\rho(x)=x^{-(1+\alpha)} L(x), x \in(0, A)$, where $L(x)$ is slowly varying at 0 and $\alpha \in(0,1)$. By Karamata's theorem, $\varphi(\varepsilon) \sim(2-\alpha)^{3 / 2}(3-\alpha)^{-1} \varepsilon^{\alpha / 2} / \sqrt{L(\varepsilon)}$ as $\varepsilon \downarrow 0$. Setting $L(x) \equiv b$, the result corresponds to the $\alpha$-stable subordinator in Example 3.1. Generalizing (4.14) to include Lévy processes on $\mathbb{R}$ (as given originally in [2]), better bounds are possible [2, p. 489]. For example, for stable processes with index $\alpha \in(0,2)$ (the Lévy measure is of the form $\left.Q(\mathrm{~d} x)=\left(a \mathbf{1}_{(-\infty, 0)}(x)+b \mathbf{1}_{(0, \infty)}(x)\right)|x|^{-(1+\alpha)} \mathrm{d} x, a, b \geq 0, a+b>0\right)$, the bound is asymptotically a constant times $\varepsilon^{\alpha / 2}$ (note the improvement compared to the subordinator case when $\alpha \in[1,2)$ ).

We are now going to consider the difference between the DFs of $X_{\varepsilon}(t) / \varepsilon$ and $X_{\star}^{c}(t)$. These are denoted by $G_{\varepsilon}(\cdot ; t)$ and $F_{\star}^{c}(\cdot ; t)$, respectively, and their difference at $x$ by $\Delta_{\star}(x ; t ; \varepsilon)$. Note that $\Delta_{\star}(x ; t ; \varepsilon)>0$ for $x>0$, by (4.2).

We begin by deriving simple bounds for $\Delta_{\star}(x ; t ; \varepsilon)$, analogous to those in (4.9) and (4.10) for $\Delta(x ; t ; \varepsilon)$. From (4.2), considering the probability that $Y_{\varepsilon}(t)$ is 0 , we obtain

$$
\Delta_{\star}(x ; t ; \varepsilon)<\left(1-\mathrm{e}^{-t \Lambda(\varepsilon)}\right) G_{\varepsilon}(x ; t), \quad \varepsilon \in(0, A),
$$

for any $X \in \mathfrak{Y}^{c, \alpha}$ and $x, t>0$. Uniformity in $x$ is established by dropping $G_{\varepsilon}(x ; t)$. Letting $z=1-\mathrm{e}^{-t \Lambda(\varepsilon)},(4.15)$ gives $G_{\varepsilon}(x ; t)<F_{\star}^{c}(x ; t)+z G_{\varepsilon}(x ; t)$. Continuing iteratively, we find that $G_{\varepsilon}(x ; t)<F_{\star}^{c}(x ; t) \sum_{k=0}^{n} z^{k}+z^{n+1} G_{\varepsilon}(x ; t)$ for all $n \in \mathbb{N}$, leading to

$$
F_{\star}^{c}(x ; t)<G_{\varepsilon}(x ; t)<\mathrm{e}^{t \Lambda(\varepsilon)} F_{\star}^{c}(x ; t) \text { for all } x, t>0 .
$$

As before, from (4.15), it follows that

$$
\Delta_{\star}(x ; t ; \varepsilon)<\min \left\{t \Lambda(\varepsilon), G_{\varepsilon}(x ; t)\right\} \leq t^{*} \Lambda(\varepsilon),
$$

where $t^{*}=t^{*}(x ; \varepsilon)$ is the unique solution $t$ of $t \Lambda(\varepsilon)=G_{\varepsilon}(x ; t)$. It is important to note that if $x$ is not too large then $F_{\star}^{c}(x ; t)$ and, hence, $G_{\varepsilon}(x ; t)$ decreases to 0 very rapidly as a function of $c t$ starting from, say, $c t=x$. (Note that $F_{\star}^{c}(x ; t)=F_{\star}^{1}(x ; c t)$.) This fact is easily confirmed numerically by evaluating $\mathrm{P}\left(X_{\star}^{c}(t) \leq x\right)$ using Monte Carlo simulations according to (1.6) with $\theta=c t$. The intuition is provided by the central limit theorem, taking into account the fact that $\mathrm{E}\left[X_{\star}^{c}(t)\right]=c t$ and $\operatorname{var}\left[X_{\star}^{c}(t)\right]=c t / 2$.

We now prove a more sophisticated estimate for $G_{\varepsilon}(x ; t)$.

Theorem 4.2. Suppose that $X \in \mathfrak{Y}^{c, \alpha}$. Define $b_{c, \alpha}(x, t)=t \mathrm{P}\left(x-U^{1 / \alpha} \leq X_{\star}^{c}(t) \leq\right.$ $x), x, t>0$, where $U$ is uniform $(0,1)$ independent of $X_{\star}^{c}(t)$. Then, for any fixed $x, t>0$,

$$
\Delta_{\star}(x ; t ; \varepsilon) \sim b_{c, \alpha}(x, t) \frac{\varepsilon^{\alpha} L(\varepsilon)}{\alpha} \quad \text { as } \varepsilon \downarrow 0 .
$$


Moreover,

$$
\sup _{x} b_{c, \alpha}(x, t) \sim \frac{\alpha}{\alpha+1} \sqrt{\frac{t}{\pi c}} \text { as } t \rightarrow \infty .
$$

Proof. Fix $x, t>0$. By (4.2),

$$
\begin{aligned}
\Delta_{\star}(x ; t ; \varepsilon) & =\mathrm{P}\left(\frac{X_{\varepsilon}(t)}{\varepsilon} \leq x\right)-\mathrm{P}\left(\frac{X_{\varepsilon}(t)}{\varepsilon}+Y_{\varepsilon}(t) \leq x\right) \\
& =\mathrm{P}\left(\frac{X_{\varepsilon}(t)}{\varepsilon} \leq x\right)-\sum_{k=0}^{\infty} \mathrm{P}\left(\frac{X_{\varepsilon}(t)}{\varepsilon}+\left(\xi_{1}+\cdots+\xi_{k}\right) \leq x\right) P_{k}(\Lambda(\varepsilon) t),
\end{aligned}
$$

where, as before, the $\xi_{i}$ are i.i.d. with distribution $J_{\varepsilon}$ (independent also of $X_{\varepsilon}(t)$ ) and the $P_{k}(\Lambda(\varepsilon) t)$ are the Poisson probabilities with mean $\Lambda(\varepsilon) t$. Hence,

$$
\Delta_{\star}(x ; t ; \varepsilon)=\Lambda(\varepsilon) t\left[\mathrm{P}\left(\frac{X_{\varepsilon}(t)}{\varepsilon} \leq x\right)-\mathrm{P}\left(\frac{X_{\varepsilon}(t)}{\varepsilon}+\xi_{1} \leq x\right)\right]+O\left(\Lambda(\varepsilon)^{2}\right) \quad \text { as } \varepsilon \downarrow 0 .
$$

Recall that $J_{\varepsilon}$ converges to the $\operatorname{beta}(\alpha, 1)$ distribution as $\varepsilon \downarrow 0$. Thus, in particular, $X_{\varepsilon}(t) / \varepsilon+$ $\xi_{1} \stackrel{\mathrm{D}}{\rightarrow} X_{\star}^{c}(t)+U^{1 / \alpha}$, with $U \sim$ uniform $(0,1)$ independent of $X_{\star}^{c}(t)$. Hence,

$$
\Delta_{\star}(x ; t ; \varepsilon)=\Lambda(\varepsilon) t\left[\mathrm{P}\left(X_{\star}^{c}(t) \leq x\right)-\mathrm{P}\left(X_{\star}^{c}(t)+U^{1 / \alpha} \leq x\right)\right]+o(\Lambda(\varepsilon)) \quad \text { as } \varepsilon \downarrow 0,
$$

and (4.16) follows. As for (4.17), let $f_{\star}^{c}(\cdot ; t)$ denote the density of $X_{\star}^{c}(t)$. If $t(\rightarrow \infty)>1 / c$, $f_{\star}^{c}(\cdot ; t)$ is continuous on $\mathbb{R}$ and continuously differentiable on $(0, \infty)\left(\right.$ since $\left.X_{\star}^{c} \in \mathfrak{S} \mathfrak{D}^{c}\right)$, and we know [18, pp. 500-501] that it attains its maximum at a (unique) point $a=a(c t) \in\left(c t-\frac{1}{2}, c t\right)-$ the mode. By [7, Theorem 2, Equation (4.2)],

$$
f_{\star}^{c}(u ; t)=\frac{1+O\left((u+c t)^{-1 / 3}\right)}{\sqrt{2 \pi c t \beta}} \exp \left[-u \xi+c t \sum_{k=1}^{\infty} \frac{\xi^{k}}{k ! k}\right]
$$

uniformly in $u \geq 1, c t \geq 1$, where $\xi$ is the unique nonzero solution of $\mathrm{e}^{\xi}-1=(u / c t) \xi$ (assuming that $u \neq c t)$ and $\beta=\mathrm{e}^{\xi}\left(\xi^{-1}-\xi^{-2}\right)+\xi^{-2}$. It follows that $f_{\star}^{c}(a ; t)$ and $f_{\star}^{c}(a-1 ; t)$ are asymptotically $1 / \sqrt{\pi c t}$ as $t \rightarrow \infty$. Finally, conditioning on $U^{1 / \alpha} \sim \operatorname{beta}(\alpha, 1)$ and then applying the mean value theorem, we obtain $b_{c, \alpha}(x, t)=t \int_{0}^{1} f_{\star}^{c}(v ; t) \alpha s^{\alpha} \mathrm{d} s$, where $v=$ $v(x, t, s) \in(x-s, x)$, from which (4.17) now straightforwardly follows.

The following result is a slight refinement of (4.16) in the case where $X$ is a gamma process.

Proposition 4.1. Suppose that $X$ is a $\Gamma_{c, \lambda}$ process. Then, for any fixed $t>0$,

$$
\Delta_{\star}(x ; t ; \varepsilon)=b_{1,1}(x, c t) \lambda \varepsilon+O\left(\varepsilon^{2}\right) \quad \text { as } \varepsilon \downarrow 0,
$$

uniformly in $x>0$.

Proof. Here $\Lambda(\varepsilon)=c \lambda \varepsilon+O\left(\varepsilon^{2}\right)$ and $\delta(s)=c\left(1-\mathrm{e}^{-\lambda s}\right)$. By (4.15), $\mathrm{P}\left(X_{\varepsilon}(t) / \varepsilon \leq\right.$ $x)-\mathrm{P}\left(X_{\star}^{c}(t) \leq x\right)$ is bounded from above by $t \Lambda(\varepsilon)$ (for all $\varepsilon>0$ ). Hence, by (4.18),

$$
\Delta_{\star}(x ; t ; \varepsilon)=c \lambda \varepsilon t \mathrm{P}\left(X_{\star}^{c}(t) \leq x\right)-\Lambda(\varepsilon) t \mathrm{P}\left(\frac{X_{\varepsilon}(t)}{\varepsilon}+\xi_{1} \leq x\right)+O\left(\varepsilon^{2}\right) \quad \text { as } \varepsilon \downarrow 0,
$$


TABLE 1: Approximation of $\sup _{x} b_{1,1}(x, t)$ (A) versus the (rounded) asymptotic counterpart $\sqrt{t /(4 \pi)}$ (B), for various values of $t$.

\begin{tabular}{cccccccccc}
\hline & \multicolumn{7}{c}{$t$} \\
\cline { 2 - 10 } & 0.25 & 0.30 & 0.40 & 0.50 & 0.75 & 1.00 & 1.75 & 2.50 & 5.00 \\
\hline A & 0.1351 & 0.1507 & 0.1772 & 0.1993 & 0.2438 & 0.2807 & 0.3722 & 0.4453 & 0.6302 \\
B & 0.1410 & 0.1545 & 0.1784 & 0.1995 & 0.2443 & 0.2821 & 0.3732 & 0.4460 & 0.6308 \\
\hline
\end{tabular}

where $\xi_{1}$ (independent of $\left.X_{\varepsilon}(t)\right)$ has distribution $J_{\varepsilon}(\mathrm{d} s)=\mathbf{1}_{(0,1]}(s) \delta(\varepsilon s) s^{-1} \Lambda(\varepsilon)^{-1} \mathrm{~d} s$. Moreover, here and in the sequel, the $O\left(\varepsilon^{2}\right)$ term is bounded in absolute value by $M \varepsilon^{2}$ for sufficiently small $\varepsilon$, where $M$ depends on $t$ (fixed) but not on $x$. By conditioning on $\xi_{1}$ and then using the bound $t \Lambda(\varepsilon)$ as above, we find that

$$
\Lambda(\varepsilon) t \mathrm{P}\left(\frac{X_{\varepsilon}(t)}{\varepsilon}+\xi_{1} \leq x\right)=c \lambda \varepsilon t \int_{0}^{1} \mathrm{P}\left(X_{\star}^{c}(t) \leq x-s\right) \mathrm{d} s+O\left(\varepsilon^{2}\right) \quad \text { as } \varepsilon \downarrow 0,
$$

from which (4.19) straightforwardly follows, noting that $b_{1,1}(x, c t)=c b_{c, 1}(x, t)$.

Remark 4.2. We can show that the $O\left(\varepsilon^{2}\right)$ term in (4.19) is asymptotically equal to

$$
\begin{aligned}
& \frac{1}{2}(c t \lambda \varepsilon)^{2}\left[\mathrm{P}\left(X_{\star}^{c}(t) \leq x\right)-2 \mathrm{P}\left(X_{\star}^{c}(t)+U \leq x\right)+\mathrm{P}\left(X_{\star}^{c}(t)+U+V \leq x\right)\right] \\
& -\frac{1}{4} c t(\lambda \varepsilon)^{2}\left[\mathrm{P}\left(X_{\star}^{c}(t) \leq x\right)-\mathrm{P}\left(X_{\star}^{c}(t)+U^{1 / 2} \leq x\right)\right],
\end{aligned}
$$

where $U$ and $V$ are i.i.d. uniform $(0,1)$, independent also of $X_{\star}^{c}(t)$. (The proof takes advantage of (4.19) itself.) The analysis of (4.20) is beyond the scope of our study.

In light of Proposition 4.1, it is worth examining the function $\psi(t):=\sup _{x} b_{1,1}(x, t)$, beyond what formula (4.17) gives. Using Monte Carlo simulations, we have concluded that this function can be approximated rather well by its asymptotic counterpart $\sqrt{t /(4 \pi)}(t \rightarrow \infty)$, quite surprisingly, starting from a relatively very small value of $t$ (with the maximum attained near $x=t)$. We have chosen certain values of $t$ in the range $[0.25,5]$, and the results are presented in Table 1. If $t$ is small, so is $\psi(t)(\leq t)$. Otherwise, the approximation $\psi(t) \cong \sqrt{t /(4 \pi)}$ keeps $\psi$ relatively small, as long as $t$ is not too large. For example, $\psi(10) \cong 0.89, \psi(50) \cong 1.99$, and $\psi(100) \cong 2.82$.

An explicit (but complicated) formula for the DF of $X_{\varepsilon}(t) / \varepsilon$ in the case where $X$ is a $\Gamma_{1, \lambda}$ process, practical for values of $x$ not far from the origin, was obtained in [4, Section 4.2]. (The adaptation to general $c>0$ is straightforward.)

To conclude this paper, we shall give a brief account of the calculation of the $\operatorname{GD}(\theta)$ distribution and density functions. Further details can be found in [4].

Let $H_{\theta}(\cdot)$ denote the $\mathrm{GD}(\theta)$ DF. Approximation of $H_{\theta}(x)$ by Monte Carlo simulations according to (1.6) may be a good idea in general. The series for $Z$ in (1.6) should be truncated at some order $M=M(\theta)$, which is increasing in $\theta$ and must be chosen appropriately with respect to the desired accuracy and computational time.

The function $H_{\theta}(x)$ can also be evaluated as a finite sum of multiple integrals. We know from the discussion of smoothness with respect to the class $\mathfrak{S} \mathfrak{D}^{c}$ that $H_{\theta}(\cdot) \in C^{\lceil\theta\rceil-1}(\mathbb{R})$ and 
$H_{\theta}^{(\lceil\theta\rceil)}(\cdot) \in C^{0}((0, \infty))$. By [4, Proposition 4.2], for all $j=0,1, \ldots,\lceil\theta\rceil$,

$$
\begin{aligned}
H_{\theta}^{(j)}(x)= & \frac{\mathrm{e}^{-\gamma \theta}}{\Gamma(\theta+1-j)} \\
& \times\left\{x^{\theta-j}+\sum_{k=1}^{\lceil x\rceil-1}(-\theta)^{k} \int_{B_{k}(x)}\left(x-\sum_{i=1}^{k} u_{i}\right)^{\theta-j} \frac{\mathrm{d} u_{1} \cdots \mathrm{d} u_{k}}{u_{1} \cdots u_{k}}\right\}, \quad x>0,
\end{aligned}
$$

where $B_{k}(x)=\left\{\boldsymbol{u} \in \mathbb{R}^{k}: 1<u_{1}<\cdots<u_{k}, u_{1}+\cdots+u_{k}<x\right\}$. A convolution form of this equation is given in [4, Equation (4.10)].

The drawback of (4.21) is obvious from a computational point of view. However, it may be quite useful if $\theta$ is relatively not large. Numerical calculations for $\theta=k / 2, k=1, \ldots, 8$, indicated that both the tail $1-H_{\theta}(x)$ and the derivatives $H_{\theta}^{(j)}(x)$ decay to 0 very rapidly for $x$ greater than some 'reasonable' value. (Asymptotic results as $x \rightarrow \infty$ can be found in [18, p. 500; Theorem 3(ii); p. 508], where $H_{\theta}$ is denoted $j_{\kappa}$.)

Theorem 1 of [6] provides a recursive algorithm useful for numerical computation of the $\mathrm{GD}(\theta)$ density function. By comparison with (4.21) (or its convolution form indicated above), it can be generalized to arbitrary $j=0,1, \ldots,\lceil\theta\rceil$ as follows:

$$
H_{\theta}^{(j)}(x)=\frac{\mathrm{e}^{-\gamma \theta} x^{\theta-j}}{\Gamma(\theta+1-j)}\left\{1+\sum_{k=1}^{\lceil x\rceil-1}(-\theta)^{k} K_{k}\left(\frac{x-k}{x-k+1} ; \theta-j\right)\right\}, \quad x>0,
$$

where $K_{k}(v ; \theta-1)=v^{\theta+k-1} \sum_{j=0}^{\infty} a_{k j} v^{j}, 0<v<1$; the coefficients $\left\{a_{k j}\right\}$ (which depend on $\theta$ ) are defined recursively in that theorem.

We further note from [4] that a result in [18, p. 497] gives, for $j=0,1, \ldots,\lceil\theta\rceil$,

$$
H_{\theta}^{(j)}(x)=x^{\theta-j}\left\{k^{j-\theta} H_{\theta}^{(j)}(k)-\theta \int_{k}^{x} z^{j-\theta-1} H_{\theta}^{(j)}(z-1) \mathrm{d} z\right\}, \quad k<x \leq k+1(k \in \mathbb{N}),
$$

thus generalizing the familiar (numerically useful) representation of the $\operatorname{GD}(\theta)$ density function, given e.g. in [1, Equation (4.26)] or [13, Equation (16)].

The formulae for $H_{\theta}^{(j)}(x)$ suggest a Taylor expansion of order $\lceil\theta\rceil-1$ around $a>0$ :

$$
H_{\theta}(x)=\sum_{j=0}^{\lceil\theta\rceil-1} \frac{H_{\theta}^{(j)}(a)}{j !}(x-a)^{j}+\frac{H_{\theta}^{(\lceil\theta\rceil)}(\xi)}{\lceil\theta\rceil !}(x-a)^{\lceil\theta\rceil}, \quad x>0,
$$

where $\xi$ is some point between $x$ and $a$. A natural choice would be $a=\theta$ (the mean). Figure 1 of [4] illustrates the practical potential of such an expansion.

\section{Acknowledgements}

My special thanks go to Mr and Mrs Shapack for funding my fellowship. I also wish to thank the anonymous referee for a detailed reading of the manuscript and valuable suggestions to shorten an earlier draft, and Professor Ely Merzbach, my MSc and PhD supervisor.

\section{References}

[1] Arratia, R., Barbour, A. D. and Tavaré, S. (2003). Logarithmic Combinatorial Structures: A Probabilistic Approach. European Mathematical Society, Zürich.

[2] Asmussen, S. AND RosińsKi, J. (2001). Approximations of small jumps of Lévy processes with a view towards simulation. J. Appl. Prob. 38, 482-493. 
[3] Bingham, N. H., Goldie, C. M. And Teugels, J. L. (1987). Regular Variation. Cambridge University Press.

[4] Covo, S. (2009). One-dimensional distributions of subordinators with upper truncated Lévy measure, and applications. Adv. Appl. Prob. 41, 367-392.

[5] Emmer, S. AND KlüPPelberg, C. (2004). Optimal portfolios when stock prices follow an exponential Lévy process. Finance Stoch. 8, 17-44.

[6] Griffiths, R. C. (1988). On the distribution of points in a Poisson Dirichlet process. J. Appl. Prob. 25, 336-345.

[7] Hensley, D. (1986). The convolution powers of the Dickman function. J. London Math. Soc. 33, 395-406.

[8] Holst, L. (2001). The Poisson-Dirichlet distribution and its relatives revisited. Preprint, Royal Institute of Technology, Stockholm. Available at http://www.math.kth.se/matstat/.

[9] Jacod, J. And Shiryaev, A. N. (2003). Limit Theorems for Stochastic Processes, 2nd edn. Springer, Berlin.

[10] Kallenberg, O. (2002). Foundations of Modern Probability, 2nd edn. Springer, New York.

[11] Kuba, M. and Panholzer, A. (2007). On weighted path lengths and distances in increasing trees. Prob. Eng. Inf. Sci. 21, 419-433.

[12] Madan, D. B., CARr, P. P. And Chang, E. C. (1998). The variance gamma process and option pricing. Europ. Finance Rev. 2, 79-105.

[13] Penrose, M. D. And Wade, A. R. (2004). Random minimal directed spanning trees and Dickman-type distributions. Adv. Appl. Prob. 36, 691-714.

[14] Sato, K.-I. (1999). Lévy Processes and Infinitely Divisible Distributions (Camb. Stud. Adv. Math. 68). Cambridge University Press.

[15] TAKÁcs, L. (1955). On stochastic processes connected with certain physical recording apparatuses. Acta Math. Acad. Sci. Hungar. 6, 363-380.

[16] Tankov, P. (2004). Lévy processes in finance: inverse problems and dependence modelling. Doctoral Thesis, École Polytechnique.

[17] VervaAt, W. (1972). Success Epochs in Bernoulli Trials (with Applications in Number Theory). Mathematisch Centrum, Amsterdam.

[18] Wheeler, F. S. (1990). Two differential-difference equations arising in number theory. Trans. Amer. Math. Soc. 318, 491-523. 